\title{
LHC signatures of warped-space vectorlike quarks
}

\author{
Shrihari Gopalakrishna, ${ }^{a}$ Tanumoy Mandal, ${ }^{a}$ Subhadip Mitra ${ }^{b}$ and Grégory Moreau ${ }^{b}$ \\ ${ }^{a}$ Institute of Mathematical Sciences (IMSc), \\ C.I.T. Campus, Taramani, Chennai 600113, India \\ ${ }^{b}$ Laboratoire de Physique Théorique, \\ CNRS-UMR 8627, Université Paris-Sud 11, \\ F-91405 Orsay Cedex, France \\ E-mail: shri@imsc.res.in, tanumoy@imsc.res.in, \\ subhadip.mitra@th.u-psud.fr, gregory.moreau@th.u-psud.fr
}

ABSTRACT: We study the LHC signatures of TeV scale vectorlike quarks $b^{\prime}, t^{\prime}$ and $\chi$ with electromagnetic charges $-1 / 3,2 / 3$ and $5 / 3$ that appear in many beyond the standard model (BSM) extensions. We consider warped extra-dimensional models and analyze the phenomenology of such vectorlike quarks that are the custodial partners of third generation quarks. In addition to the usually studied pair-production channels which depend on the strong coupling, we put equal emphasis on single production channels that depend on electroweak couplings and on electroweak symmetry breaking induced mixing effects between the heavy vectorlike quarks and standard model quarks. We identify new promising $g g$-initiated pair and single production channels and find the luminosity required for discovering these states at the LHC. For these channels, we propose a cut that allows one to extract the relevant electroweak couplings. Although the motivation is from warped models, we present many of our results model-independently.

Keywords: Phenomenology of Field Theories in Higher Dimensions, Hadronic Colliders

ArXiv EPRINT: 1306.2656 


\section{Contents}

1 Introduction 1

2 Warped models $\quad 4$

2.1 Model without $Z b \bar{b}$ protection (DT model) 5

2.2 Model with $Z b \bar{b}$ custodial protection 8

2.2.1 Model with $t_{R}=(1,1)_{2 / 3}$ (ST model) $\quad 9$

2.2.2 Model with $t_{R} \subset(1,3)_{2 / 3} \oplus(3,1)_{2 / 3}$ (TT model) 11

3 Parameters and couplings $\quad 12$

$\begin{array}{ll}3.1 \chi \text { parameters and couplings } & 13\end{array}$

$\begin{array}{lll}3.2 t^{\prime} \text { parameters and couplings } & 15\end{array}$

$\begin{array}{lll}3.3 & b^{\prime} \text { parameters and couplings } & 15\end{array}$

4 Decay width and branching ratio 18

$5 \quad$ LHC signatures $\quad 22$

$5.1 \chi$ LHC signatures $\quad 22$

$\begin{array}{ll}5.2 t^{\prime} \text { LHC signatures } & 27\end{array}$

$5.3 b^{\prime}$ LHC signatures $\quad 31$

6 Conclusions $\quad 34$

$\begin{array}{ll}\text { A Fermion profiles } & 35\end{array}$

B $t_{R}$ triplet case diagonalization $\quad 36$

$\begin{array}{ll}\text { C } \chi \text { signature in more detail } & 37\end{array}$

\section{Introduction}

The standard model (SM) of particle physics suffers from the gauge hierarchy and flavor hierarchy problems and many beyond the standard model (BSM) extensions have been proposed to solve these problems. The extra particles in these BSM extensions are being searched for at the CERN Large Hadron Collider (LHC). Some BSM extensions contain vectorlike colored fermions, for example, having electromagnetic (EM) charges 5/3, 2/3, and $-1 / 3$, which we denote as $\chi, t^{\prime}$ and $b^{\prime}$ respectively. For instance, warped-space extradimensional models with bulk fermions contain these vectorlike fermions.

In this work we consider the LHC signatures of the $\chi, t^{\prime}$ and $b^{\prime}$. We present a few warped-space extra-dimensional models that contain these states, specify realistic parameter values, and extract the couplings of these vectorlike fermion states with SM states. We 
identify promising pair and single production channels at the LHC and find the luminosity required for discovering these states at the LHC. We emphasize that the signatures we identify and the search strategies are common to many other BSM theories that contain such vectorlike quarks. A particular emphasis is the single-production of the heavy colored fermions in addition to their pair-production, since single-production couplings depend more directly on their electroweak quantum numbers, while pair-production is dominated by its coupling to the gluon which is given by the $\mathrm{SU}(3)_{C}$ gauge coupling $g_{s}$, and thus hides its electroweak aspects. Although measuring the branching ratios using pair-production channels gives information on the electroweak couplings, it is only the ratios of couplings that is determined and not their actual values. But single-production can fix the actual values of the couplings. Moreover, compared to pair-production, single production also have less complications from combinatorics. Depending on the coupling, some single production channel can even be the dominant production channel for heavy vectorlike quarks due to the phase-space suppression in pair-production.

In ref. [1], we analyzed the LHC signatures of a vectorlike $b^{\prime}$ in a model-independent fashion. We highlighted there many general aspects of vectorlike fermions and contrasted them with chiral (4th generation) fermions in how they decay and their resulting signatures at the LHC. In this work we extend this to include the $\chi$ and $t^{\prime}$ also.

Other than in extra dimensional theories, vectorlike quarks appear in many new-physics models such as composite Higgs models [2-5], little Higgs models [6-9], some supersymmetric extensions [10-12], quark-lepton unification models [13] etc. Extensive studies on vectorlike fermions are available in the literature. Here we briefly survey some references that are relevant to our study. Vectorlike fermions in the context of Higgs boson production have been considered in refs. [14-18]. Based on the recent discovery of a Higgs boson at the LHC [19, 20], refs. [21, 22] constrain vectorlike fermion masses and couplings from the recent data. It has been pointed out [23-26] that vectorlike fermions can address the forwardbackward asymmetry in top quark pair production at the Tevatron. Refs. [27-33] analyze vectorlike fermion representations and mixing of the new fermions with the SM quarks and the relevant experimental bounds. Refs. [34-42] study the LHC signatures of $b^{\prime}, t^{\prime}$ and $\chi$ vectorlike quarks. Ref. [36] studies the LHC signatures of vectorlike $b^{\prime}$ and $\chi$ in the $4-W$ channel. Ref. [41] studies multi- $b$ signals for $t^{\prime}$ quarks. The LHC signatures of vectorlike $t^{\prime}$ and $b^{\prime}$ decaying to a Higgs boson are discussed in ref. [40]. Ref. [42] studies pair-production of the vectorlike quarks followed by decays into single and multi-lepton channels and the pair-production of the Kaluza-Klein (KK) top is explored in ref. [43]. Ref. [44] studies the signatures of vectorlike quarks resulting from the decay of a KK gluon. Ref. [45] analyzes the single production of $t^{\prime}$ and $b^{\prime}$ via KK gluon and finds that these channels could be competitive with the direct electroweak single production channels of these heavy quarks. Model independent LHC searches of vectorlike fermions have been discussed in refs. [4649]. Many important pair and single production channels for probing a vectorlike $b^{\prime}$ at the LHC in the context of a warped extra-dimension were explored in ref. [50]. Mixing of the SM $b$-quark with a heavy vectorlike $b^{\prime}$ and partial decay widths were worked out in ref. [51]. In ref. [52], the LHC phenomenology of new heavy chiral quarks with electric charges $-4 / 3$ and 5/3 are discussed. Exploiting same-sign dileptons signal to beat the SM background, 
refs. $[34,35]$ show that the pair-production at the $14 \mathrm{TeV}$ LHC can discover charge $-1 / 3$ and $5 / 3$ vectorlike quarks with a mass up to $1 \mathrm{TeV}(1.5 \mathrm{TeV})$ with about $10 \mathrm{fb}^{-1}\left(200 \mathrm{fb}^{-1}\right)$ integrated luminosity. Ref. [37] considers pair production of charge $5 / 3$ vectorlike quarks and shows that with the search for same sign dilepton the discovery reach of the $7 \mathrm{TeV}$ LHC is about $700 \mathrm{GeV}$ with $5 \mathrm{fb}^{-1}$ integrated luminosity. The LHC signatures of $t^{\prime}$ vectorlike quarks have been discussed in [38] using $p p \rightarrow t^{\prime} \bar{t}^{\prime} \rightarrow b W^{+} \bar{b} W^{-}$channel with the semileptonic decay of the $W$ 's and the reach is found to be about $1 \mathrm{TeV}$ with $100 \mathrm{fb}^{-1}$ integrated luminosity at the $14 \mathrm{TeV}$ LHC. With $14.3 \mathrm{fb}^{-1}$ of integrated luminosity at the $8 \mathrm{TeV}$ LHC, ATLAS has excluded a weak-isospin singlet $b^{\prime}$ quark with mass below $645 \mathrm{GeV}$, while for the doublet representation the limit is $725 \mathrm{GeV}$ [53]. With $4.64 \mathrm{fb}^{-1}$ luminosity, using single production channels with charged and neutral current interactions, vectorlike $b^{\prime}, t^{\prime}$ and $\chi$ quarks up to masses about $1.1 \mathrm{TeV}, 1 \mathrm{TeV}$ and $1.4 \mathrm{TeV}$ respectively have been excluded [54], for couplings taken to be $v / M$, where $v$ is the Higgs vacuum expectation value (VEV), and $M$ the mass of the vectorlike quark. In ref. [55] the CMS collaboration presents the results for the search of a charge $5 / 3$ quark at the $7 \mathrm{TeV}$ LHC. With $5 \mathrm{fb}^{-1}$ luminosity and assuming $100 \%$ branching ratio (BR) for the $\chi \rightarrow t W$ channel a $\chi$ quark with mass below $645 \mathrm{GeV}$ is excluded. With the $8 \mathrm{TeV}$ LHC, the CMS collaboration has improved their limit on the $\chi$ quark to $770 \mathrm{GeV}$ [56]. In ref. [57] the ATLAS collaboration shows the exclusion limits for a $t^{\prime}$ quark in the $\operatorname{BR}\left(t^{\prime} \rightarrow W b\right)$ versus $\operatorname{BR}\left(t^{\prime} \rightarrow t h\right)$ plane.

In this work, we detail some warped models with different $\mathrm{SU}(2)_{L} \otimes \mathrm{SU}(2)_{R}$ fermion representations that have been proposed earlier in the literature. For each of these we carefully work out the couplings induced by electroweak symmetry breaking (EWSB) relevant for single production of the vectorlike quarks after diagonalizing the mass matrices including the EWSB contributions. We show what sizes of the relevant couplings are realistic by varying the parameters of the theory. For these warped models with the couplings above, and for vectorlike quark masses of about a $\mathrm{TeV}$, the direct single production channels that most of the studies above focus on have too small cross-sections and therefore extraction of the electroweak couplings from these are difficult. Typically these quark initiated processes have small rates. In this work, we identify channels which are $g g$ initiated but yet sensitive to electroweak couplings after our cuts. For vectorlike quark masses of about a $\mathrm{TeV}$, the channels are signal rate limited and the backgrounds under control after cuts. We show that these channels can be observed above background. These are our main contributions.

This paper is organized as follows: in section 2 we give the details of the warped models both with and without custodial protection of the $Z b \bar{b}$ coupling, show the mass mixing terms and their diagonalization, and work out the couplings in the mass basis relevant to the phenomenology we consider. In A we give the fermion profiles that we use to compute the couplings, and the dependence of the mass eigenvalue on the $c$-parameter that parametrizes the fermion bulk masses in units of the curvature scale of the extradimension. In $\mathrm{B}$ we give some analytical results of the diagonalization and the resulting couplings in the small mixing limit for the model with custodial protection of $Z b \bar{b}$. In section 3 we give details of the parameter choices we make in the warped models and show the vectorlike fermion couplings and their dependence on the $c$-parameter. Readers not wanting to know all the details of the warped models can go directly to the next section, 
although the above sections will guide which channels we consider in later sections. In section 4 we give the decay partial widths and the branching ratios into the various decay modes. In section 5 we discuss some promising discovery channels for the vectorlike quarks and present the reach for the 8 and $14 \mathrm{TeV}$ LHC. We offer our conclusions in section 6 .

\section{$2 \quad$ Warped models}

The Randall-Sundrum model [58] is a theory defined on a slice of AdS space which solves the gauge hierarchy problem. Due to the AdS/CFT duality conjecture [59], this construction may be dual to a spontaneously broken conformal four dimensional strongly coupled theory. By letting SM fields propagate in the bulk, the fermion mass hierarchy of the SM can also be addressed [60, 61] without badly violating the flavor-changing neutral current (FCNC) constraints. The bulk mass parameters $c_{\psi}$ are chosen so that the SM fermion masses match the measured values.

Precision electroweak constraints place strong bounds on such extensions of the SM. Gauging $\mathrm{SU}(2)_{R}$ in the bulk offers a custodial symmetry that protects [62] the $T$-parameter from receiving large tree-level shifts, but can still lead to problems due to an excessive shift to the $Z b \bar{b}$ coupling. This can also be protected [63] by taking the third generation $Q_{L}$ as a bi-doublet under $\mathrm{SU}(2)_{L} \times \mathrm{SU}(2)_{R}$, i.e. $Q_{L}=(2,2)$.

An equivalent $4 D$ theory can be written down by performing a KK expansion. For LHC phenomenology, it is sufficient to keep only the zero-mode and the 1st KK excitation with mass $M_{K K}$. EWSB makes some zero-modes massive like in the SM, and mixes various KK modes, and after diagonalization the light eigenmodes are identified with the SM states. In this work, we ignore mixings between zero-mode and 1st KK modes in the gauge sector as this mixing is of order $\sqrt{k \pi R} v^{2} / M_{K K}^{2}$ and will be a few percent effect. We keep the $(0)-(1)$ mixing in the fermion sector to fermions with Dirichlet-Neumann $(-,+)$ boundary conditions $(\mathrm{BC})$ as these can be bigger owing to the smaller mass of the $(-,+)$ custodians.

The $\mathrm{SU}(2)_{R}$ symmetry implies extra exotic $5 \mathrm{D}$ fermions not present in the $\mathrm{SM}$, and the light zero-modes of these which are not observed in Nature are "projected-out" by imposing $(-,+) \mathrm{BC}$ on the bulk fields. The first KK excitation of such $(-,+)$ fermions, i.e. the custodial partners, especially of third generation quarks can be significantly lighter [62, 64$66]$ than the gauge KK excitations, leading to measurable signals at the LHC.

In warped space extra-dimensional theories, in order to relax electroweak constraints, $\mathrm{SU}(2)_{R}$ is gauged in the bulk [62] to provide a custodial symmetry in the gauge-Higgs sector that protects the $T$ parameter. We therefore take the bulk gauge group as $\mathrm{SU}(2)_{\mathrm{L}} \times \mathrm{SU}(2)_{\mathrm{R}} \times \mathrm{U}(1)_{\mathrm{X}}$. We start with the simplest realization of this in section 2.1 although the constraint coming from the shift of the $Z b \bar{b}$ coupling is quite strong. In order to avoid this constraint, the $\mathrm{SU}(2)_{R}$ can also be used to protect this coupling [63], and we present this model in section 2.2. The most important aspect, as already pointed out, is that the new heavy fermions (the first KK fermion modes in particular) are vectorlike with respect to the gauge group. In this work we focus on the LHC signatures of three such custodial vectorlike quarks, namely the $\chi, t^{\prime}$ and $b^{\prime}$. This complements other studies 
of warped KK states at the LHC, for example, KK graviton in ref. [67] and KK Gauge bosons in refs. [68-73].

Following usual practice, we denote the field representations as $(l, r)_{X}$ where $l, r$ denote the $\mathrm{SU}(2)_{L}$ and $\mathrm{SU}(2)_{R}$ representations respectively, and $X$ denotes the $\mathrm{U}(1)_{X}$ charge. In all the Lagrangian terms in the following, we will not show terms that are the same as in the SM, but will only show the terms either new to this BSM theory, or SM couplings that are shifted.

\subsection{Model without $Z b \bar{b}$ protection (DT model)}

We start with the quark representations

$$
\begin{aligned}
Q_{L} & \equiv(\mathbf{2}, \mathbf{1})_{1 / 6}=\left(t_{L}, b_{L}\right), \\
\Psi_{b_{R}} & \equiv(\mathbf{1}, \mathbf{2})_{1 / 6}=\left(t^{\prime}, b_{R}\right), \quad \Psi_{t_{R}} \equiv(\mathbf{1}, \mathbf{2})_{1 / 6}=\left(t_{R}, b^{\prime}\right) .
\end{aligned}
$$

The representation for the Higgs field, responsible for the EWSB, is

$$
\Sigma \equiv(\mathbf{2}, \mathbf{2})_{0}
$$

We refer to this model as the doublet-top (DT) model. The extra fields $t^{\prime}$ and $b^{\prime}$ (the "custodians") are ensured to be without zero-modes by applying Dirichlet-Neumann $(-,+)$ $\mathrm{BC}$ on the extra dimensional interval $[0, \pi R]$, and their KK excitations are vectorlike with respect to the SM gauge group, while the SM particles are the zero-modes of fields with Neumann-Neumann $(+,+) \mathrm{BC}$, and are chiral. As mentioned above, the $(-,+)$ fields are most likely the lowest mass KK excitation, and, among them the $b^{\prime}$ couplings to SM states are larger due to a larger mixing angle. This is because the mixing angle is inversely proportional to $M_{b^{\prime}}$ which is smaller due to the $c_{t_{R}}$ choice required for the correct topquark mass. Therefore, the $b^{\prime}$ promises to have the best observability at the LHC, and we will only study its phenomenology and will not comment further on the $t^{\prime}$ for this model. Elsewhere in the literature, sometimes the $L, R$ subscripts on fermion fields denote the gauge-group, but in our notation here, the $b_{L, R}^{\prime}$ will mean the two Lorentz chiralities of the vectorlike $b^{\prime}$.

Electroweak symmetry is broken by $\langle\Sigma\rangle=\operatorname{diag}(v, v) / \sqrt{2}$ (the Higgs boson VEV is $v \approx 246 \mathrm{GeV})$. The Goldstone bosons of electroweak symmetry breaking $(\phi)$ are contained in $\Sigma=(v / \sqrt{2}) e^{2 i \phi^{a} T^{a} / v}$, written in the nonlinear realization, where $T^{a}$ are the generators of $\mathrm{SU}(2)_{L}$. We work here in the unitary gauge for which we absorb the Goldstone bosons as the longitudinal polarization of the gauge bosons. Nevertheless, for completeness and to have a clear understanding of the couplings involved, a derivation of the couplings using Goldstone boson equivalence is presented in appendix A of ref. [50]. The theory as written above has also been presented before in ref. [50].

The Yukawa couplings are given by

$$
\mathcal{L}_{5 D} \supset-\lambda_{t} \bar{Q}_{L} \Sigma \Psi_{t_{R}}-\lambda_{b} \bar{Q}_{L} \Sigma \Psi_{b_{R}}
$$

where $\lambda_{t, b}$ are the $5 \mathrm{D}$ Yukawa coupling constants. We write down an equivalent $4 D$ theory by a Kaluza-Klein expansion. After EWSB, the zero-mode $b$ mixes with the $b^{\prime}$ due to 
off-diagonal terms in the following mass matrix:

$$
\mathcal{L}_{4 D} \supset-\left(\begin{array}{ll}
\overline{b_{L}} & b^{\top}
\end{array}\right)\left(\begin{array}{cc}
\lambda_{Q_{L} b_{R}} v / \sqrt{2} & \lambda_{Q_{L} b_{R}^{\prime}} v / \sqrt{2} \\
\lambda_{b_{R} b_{L}^{\prime}} v / \sqrt{2} & M_{b^{\prime}}
\end{array}\right)\left(\begin{array}{c}
b_{R} \\
b^{\prime}{ }_{R}
\end{array}\right)+\text { h.c. },
$$

where $\lambda_{Q_{L} b_{R}}$ is the zero-mode b-quark Yukawa coupling, the $M_{b^{\prime}}$ is the vectorlike mass, and $\lambda_{i j} v / \sqrt{2}$ terms are induced after EWSB. In this work we set $\lambda_{b_{R} b_{L}^{\prime}}$ to zero since this will always be the case we are interested in.

The above mass matrix written in the $\left(b, b^{\prime}\right)$ basis is diagonalized by bi-orthogonal rotations, and we denote the sine (cosine) of the mixing angles by $s_{\theta}^{L, R}\left(c_{\theta}^{L, R}\right)$. We denote the corresponding mass eigenstates as $\left(b_{1}, b_{2}\right)$. We define the off-diagonal mass $\tilde{m} \equiv \lambda_{Q_{L} b_{R}^{\prime}} v / \sqrt{2}$ for notational ease. The mixing angles are

$$
\tan \left(2 \theta_{L}\right)=-\frac{2 \tilde{x}}{\left(1-\tilde{x}^{2}-x_{b}^{2}\right)} ; \quad \tan \left(2 \theta_{R}\right)=-\frac{2 x_{b} \tilde{x}}{\left(1+\tilde{x}^{2}-x_{b}^{2}\right)}
$$

where $x_{b} \equiv\left(\lambda_{Q_{L} b_{R}} v / \sqrt{2}\right) / M_{b^{\prime}}$ and $\tilde{x} \equiv \tilde{m} / M_{b^{\prime}}$. The mass eigenvalues to leading order in $x_{b}$ are:

$\left(\lambda_{Q_{L} b_{R}} v / \sqrt{2}\right) / \sqrt{1+\tilde{x}^{2}}$ and $M_{b^{\prime}} \sqrt{\left(1+\tilde{x}^{2}\right)\left(1+x_{b}^{2} \tilde{x}^{2} /\left(1+\tilde{x}^{2}\right)^{2}\right)}$. Although we do not show the mass matrix for the top sector, analogously, the top mass is given by $m_{t} \approx \lambda_{Q_{L} t_{R}} v / \sqrt{2}$.

The $b^{\prime}$ mixes with the zero-mode $b$ due to off-diagonal terms in the mass matrix induced by EWSB as shown in eq. (2.2). Diagonalizing this, we go from the $\left(b, b^{\prime}\right)$ basis to the $\left(b_{1}, b_{2}\right)$ mass-basis and write an effective Lagrangian relevant for this model in the mass-basis as [1]

$$
\begin{aligned}
\mathcal{L}_{4 D} \supset & -\frac{e}{3} \overline{b_{1}} \gamma^{\mu} b_{1} A_{\mu}-\frac{e}{3} \bar{b}_{2} \gamma^{\mu} b_{2} A_{\mu}+g_{s} \overline{b_{1}} \gamma^{\mu} T^{\alpha} b_{1} g_{\mu}^{\alpha}+g_{s} \bar{b}_{2} \gamma^{\mu} T^{\alpha} b_{2} g_{\mu}^{\alpha} \\
& -\left(\kappa_{b t W}^{L} \overline{t_{L}} \gamma^{\mu} b_{1 L} W_{\mu}^{+}+\kappa_{b_{2} t W}^{L} \bar{t}_{1 L} \gamma^{\mu} b_{2 L} W_{\mu}^{+}+\text {h.c. }\right) \\
& +\kappa_{b b Z}^{L} \overline{b_{1 L}} \gamma^{\mu} b_{1 L} Z_{\mu}+\kappa_{b_{2} b_{2} Z}^{L} \bar{b}_{2 L} \gamma^{\mu} b_{2 L} Z_{\mu} \\
& +\left(\kappa_{b_{2} b Z}^{L} \bar{b}_{1 L} \gamma^{\mu} b_{2 L} Z_{\mu}+\text { h.c. }\right) \\
& +\kappa_{b b Z}^{R} \overline{b_{1 R}} \gamma^{\mu} b_{1 R} Z_{\mu}+\kappa_{b_{2} b_{2} Z}^{R} \bar{b}_{2 R} \gamma^{\mu} b_{2 R} Z_{\mu}
\end{aligned}
$$

and the Higgs interactions as $[1]^{1}$

$$
\begin{aligned}
\mathcal{L}_{4 D} \supset & -h\left[\kappa_{h b_{L} b_{R}} \bar{b}_{1 L} b_{1 R}+\kappa_{h b_{2 L} b_{2 R}} \bar{b}_{2 L} b_{2 R}\right. \\
& \left.+\kappa_{h b_{L} b_{2 R}} \bar{b}_{1 L} b_{2 R}+\kappa_{h b_{2 L} b_{R}} \bar{b}_{2 L} b_{1 R}\right]+ \text { h.c. }
\end{aligned}
$$

We have not introduced $\kappa_{b_{2} b Z}^{R} \bar{b}_{2 R} \gamma^{\mu} b_{1 R} Z_{\mu}+$ h.c. or $\kappa_{b_{2} t W}^{R} \bar{t}_{1 R} \gamma^{\mu} b_{2 R} W_{\mu}^{+}+$h.c. in eq. (2.4) since these couplings will not arise in this model. For convenience, we use $b$ and $b_{1}$ interchangeably, and also $b^{\prime}$ and $b_{2}$ interchangeably, but it should be clear from the context which one we mean.

\footnotetext{
${ }^{1}$ Our convention of the Higgs coupling $\kappa$ 's here differ by a factor of $\sqrt{2}$ compared to that in ref. [1].
} 
For mixing with a single $b^{\prime}$, the effective couplings $\kappa$ as defined in eqs. (2.4) and (2.5) are given by

$$
\begin{aligned}
\kappa_{b t W}^{L} & =\frac{g c_{\theta}^{L}}{\sqrt{2}} ; & \kappa_{b_{2} t W}^{L} & =\frac{g s_{\theta}^{L}}{\sqrt{2}} ; \\
\kappa_{b b Z}^{L} & =g_{Z}\left(-\frac{1}{2} c_{\theta}^{L}+\frac{1}{3} s_{W}^{2}\right) ; & \kappa_{b_{2} b_{2} Z}^{L} & =g_{Z}\left(-\frac{1}{2} s_{\theta}^{L}+\frac{1}{3} s_{W}^{2}\right) ; \\
\kappa_{b_{2} b Z}^{L} & =g_{Z} c_{\theta}^{L} s_{\theta}^{L}\left(\frac{1}{2}\right) ; \quad \kappa_{b b Z}^{R}=g_{Z}\left(\frac{1}{3} s_{W}^{2}\right) ; & \kappa_{b_{2} b_{2} Z}^{R} & =g_{Z}\left(\frac{1}{3} s_{W}^{2}\right) ; \\
\kappa_{h b_{L} b_{R}} & =\frac{1}{\sqrt{2}}\left(c_{\theta}^{L} c_{\theta}^{R} \lambda_{Q_{L} b_{R}}+c_{\theta}^{L} s_{\theta}^{R} \lambda_{Q_{L} b_{R}^{\prime}}\right) ; & \kappa_{h b_{2 L} b_{2 R}} & =\frac{1}{\sqrt{2}}\left(s_{\theta}^{L} s_{\theta}^{R} \lambda_{Q_{L} b_{R}}-s_{\theta}^{L} c_{\theta}^{R} \lambda_{Q_{L} b_{R}^{\prime}}\right) ; \\
\kappa_{h b_{L} b_{2 R}} & =\frac{1}{\sqrt{2}}\left(-c_{\theta}^{L} s_{\theta}^{R} \lambda_{Q_{L} b_{R}}+c_{\theta}^{L} c_{\theta}^{R} \lambda_{Q_{L} b_{R}^{\prime}}\right) ; & \kappa_{h b_{2 L} b_{R}} & =\frac{1}{\sqrt{2}}\left(-s_{\theta}^{L} c_{\theta}^{R} \lambda_{Q_{L} b_{R}}-s_{\theta}^{L} s_{\theta}^{R} \lambda_{Q_{L} b_{R}^{\prime}}\right) .
\end{aligned}
$$

From eq. (2.6) we see that $\kappa_{b b Z}^{L}$ is shifted, and experimental constraints require that this shift be less than about $1 \%$, roughly implying $s_{\theta}^{L}<0.1$, i.e. $M_{b^{\prime}} \gtrsim 10 \tilde{m} \approx 3 \mathrm{TeV}$. But as we have mentioned, since we have in mind application to the model in ref. [63] where this coupling is protected by the custodial symmetry, we consider much lighter $M_{b^{\prime}}$ when we discuss the phenomenology.

For this model, the effective $4 D$ Yukawa couplings parametrized in eq. (2.2) are given by

$$
\begin{aligned}
\lambda_{b_{R} b_{L}^{\prime}}=0 ; & \lambda_{Q_{L} b_{R}}=\frac{\tilde{\lambda}_{b}}{k \pi R} f_{Q_{L}}(\pi R) f_{b_{R}}(\pi R) e^{k \pi R} ; \\
\lambda_{Q_{L} b_{R}^{\prime}}=\frac{\tilde{\lambda}_{t}}{k \pi R} f_{Q_{L}}(\pi R) f_{b_{R}^{\prime}}(\pi R) e^{k \pi R} ; & \lambda_{Q_{L} t_{R}}=\frac{\tilde{\lambda}_{t}}{k \pi R} f_{Q_{L}}(\pi R) f_{t_{R}}(\pi R) e^{k \pi R},
\end{aligned}
$$

where $\lambda_{Q_{L} b_{R}}$ is the b-quark Yukawa coupling, $\lambda_{Q_{L} t_{R}}$ is the top-quark Yukawa coupling, $\tilde{\lambda}_{b, t}$ are the (dimensionless) $5 D$ Yukawa couplings $\tilde{\lambda}_{b, t} \equiv k \lambda_{b, t}$, and $f_{\psi}$ are the fermion wavefunctions which depend on the fermion bulk mass parameters $c_{\psi}$ [61]. We present the fermion profiles in A.

The mixing in the gauge boson sector i.e., $V_{\mu}^{(0)} \leftrightarrow V_{\mu}^{(1)}$ mixing, where $V_{\mu}=\left\{W_{\mu}, Z_{\mu}\right\}$, also induces the $W t b^{\prime}$ coupling, and this mixing is of order $\left(v / M_{K K}\right)^{2}$ with an additional $\sqrt{k \pi R}$ enhancement for an IR-brane-peaked Higgs. The contribution to the $b^{\prime}$ decay rate due to $b^{\prime} \leftrightarrow b$ mixing is proportional to $\left(\tilde{m} / M_{b^{\prime}}\right)^{2}$, while due to $W_{L}^{(0)} \leftrightarrow W_{R}^{(1)}$ mixing it is proportional to $\left(\sqrt{k \pi R}\left(g_{R} / g_{L}\right) m_{W}^{2} / M_{W_{R}^{\prime}}^{2}\right)^{2}$, and it should be noted that the gauge KK boson mass (i.e. $M_{W_{R}^{\prime}}$ ) is constrained to be $\gtrsim 2 \mathrm{TeV}$ by precision electroweak constraints (see ref. [74] and references therein). Thus, the contribution due to gauge KK mixing is about $1.3 \%$ of the fermion KK mixing contribution for $M_{b^{\prime}}=M_{W_{R}^{\prime}}=2 \mathrm{TeV}$, and even smaller for lighter $b^{\prime}$ masses. We thus do not include the $W^{(0)} \leftrightarrow W^{(1)}$ mixing contribution in our study. See ref. [36] for another discussion of the $W^{(0)} \leftrightarrow W^{(1)}$ mixing contribution. For the model without custodial protection of $Z b b$, we ignore $t \leftrightarrow t^{\prime}$ mixing since this mixing angle is small, being suppressed by the larger $M_{t^{\prime}}$ (above $3 \mathrm{TeV}$ ) due to the choice of the $c_{b_{R}}$ required for the correct $b$-quark mass. We also ignore mixings to the heavier KK modes in both the gauge and fermion sectors. 


\subsection{Model with $Z b \bar{b}$ custodial protection}

In order to ease precision electroweak constraints on warped models, the custodial symmetry can be used to protect the $Z b \bar{b}$ coupling as proposed in ref. [63]. One way to achieve this is to complete the $3 \mathrm{rd}$ generation left-handed quarks into the $Q_{L}=(2,2)_{2 / 3}$ bi-doublet representation and the theory made invariant under a discrete $L \leftrightarrow R$ symmetry defined as $P_{L R}$. The kinetic energy (KE) term for $Q_{L}$ is

$$
\mathcal{L}_{\mathrm{KE}} \supset \operatorname{Tr}\left[\bar{Q}_{L} i \gamma^{\mu} D_{\mu} Q_{L}\right],
$$

and $\Sigma=(2,2)_{0}$ is the bidoublet Higgs, and their component fields are

$$
Q_{L}=\left(\begin{array}{cc}
t_{L} & \chi \\
b_{L} & t^{\prime}
\end{array}\right), \quad \Sigma=\left(\begin{array}{cc}
\phi_{0}^{*} & \phi^{+} \\
-\phi^{-} & \phi_{0}
\end{array}\right) .
$$

EWSB is due to $\left\langle\phi_{0}\right\rangle=v / \sqrt{2}$ and $\operatorname{Im}\left(\phi^{a}\right)$ are the Goldstone bosons. Note that to complete the bidoublet representation, two new fermions have been introduced, namely $\chi$ and $t^{\prime}$, with electromagnetic charge $5 / 3$ and $2 / 3$ respectively. The extra-fields $\chi$ and $t^{\prime}$ (the "custodians") are ensured to be without zero-modes by applying Dirichlet-Neumann $(-,+)$ boundary conditions $(\mathrm{BC})$ on the extra dimensional interval $[0, \pi R]$, and their KK excitations are vectorlike with respect to the SM gauge group, while the SM particles are the zero-modes of fields with Neumann-Neumann $(+,+)$ BC, and are chiral. Elsewhere in the literature, sometimes the $L, R$ subscripts on fermion fields denote the gauge-group, but in our notation, the subscripts $L, R$ on the fields denote the left and right (Lorentz) chiralities.

The above $\mathcal{L}$ in eqs. (2.8) implies the following couplings of the component fields

$$
\begin{aligned}
\mathcal{L}_{\mathrm{KE}} \supset & \frac{g_{L}}{2} W_{L \mu}^{3}\left[\bar{t}_{L} \gamma^{\mu} t_{L}-\bar{b}_{L} \gamma^{\mu} b_{L}+\bar{\chi} \gamma^{\mu} \chi-\bar{t}^{\prime} \gamma^{\mu} t^{\prime}\right]+\frac{g_{L}}{\sqrt{2}}\left[W_{L \mu}^{+}\left(\bar{t}_{L} \gamma^{\mu} b_{L}+\bar{\chi} \gamma^{\mu} t^{\prime}\right)+\text { h.c. }\right] \\
& +g^{\prime} B_{\mu}\left[\frac{1}{6} \bar{t}_{L} \gamma^{\mu} t_{L}+\frac{1}{6} \bar{b}_{L} \gamma^{\mu} b_{L}+\frac{7}{6} \bar{\chi} \gamma^{\mu} \chi+\frac{7}{6} \bar{t}^{\prime} \gamma^{\mu} t^{\prime}\right] .
\end{aligned}
$$

The $\mathrm{SU}(3)_{c}$ QCD interaction of the colored fermions are standard and are not shown.

We go to the electroweak gauge boson mass basis by the usual orthogonal rotation

$$
\left(\begin{array}{c}
B \\
W_{L}^{3}
\end{array}\right)=\left(\begin{array}{cc}
c_{W} & -s_{W} \\
s_{W} & c_{W}
\end{array}\right)\left(\begin{array}{c}
A \\
Z
\end{array}\right),
$$

defined by the weak mixing angle $c_{W} \equiv \cos \left(\theta_{W}\right)=g_{L} / \sqrt{g_{L}^{2}+g^{\prime 2}}, s_{W} \equiv \sin \left(\theta_{W}\right)=$ $g^{\prime} / \sqrt{g_{L}^{2}+g^{\prime 2}}$, and the electric charge as $e \equiv g_{L} g^{\prime} / \sqrt{g_{L}^{2}+g^{\prime 2}}$.

After KK reduction, we obtain, in addition to the SM neutral current (NC) and charge current $(\mathrm{CC})$ interactions, the following new interactions

$$
\begin{aligned}
\mathcal{L}_{\mathrm{NC}}^{4 D} \supset \mathcal{L}_{\mathrm{NC}}^{S M}+\left[e \mathcal{I}_{\chi \chi A}\left(\frac{5}{3}\right) A_{\mu}+g_{Z} \mathcal{I}_{\chi \chi Z}\left(\frac{1}{2}-s_{W}^{2} \frac{5}{3}\right) Z_{\mu}\right] \bar{\chi} \gamma^{\mu} \chi \\
+\left[e \mathcal{I}_{t^{\prime} t^{\prime} A}\left(\frac{2}{3}\right) A_{\mu}+g_{Z} \mathcal{I}_{t^{\prime} t^{\prime} Z}\left(-\frac{1}{2}-s_{W}^{2} \frac{2}{3}\right) Z_{\mu}\right] \overline{t^{\prime}} \gamma^{\mu} t^{\prime} \\
\mathcal{L}_{\mathrm{CC}}^{4 D} \supset \mathcal{L}_{\mathrm{CC}}^{S M}+\frac{g_{L}}{\sqrt{2}} \mathcal{I}_{\chi t^{\prime} W} W_{L \mu}^{+} \bar{\chi} \gamma^{\mu} t^{\prime}+\text { h.c. }
\end{aligned}
$$


where $g_{Z}=\sqrt{g_{L}^{2}+g^{\prime 2}}$, and the overlap integrals are given by

$$
\mathcal{I}_{\psi \psi V} \equiv \frac{1}{\pi R} \int_{0}^{\pi R} d y e^{k y} f_{\psi}(y) f_{\psi}(y) f_{V}(y) .
$$

Since $\mathrm{U}(1)_{E M}$ is unbroken $\mathcal{I}_{\psi \psi A}=1 ; \mathcal{I}_{\psi \psi Z}$ and $\mathcal{I}_{\psi \psi W}$ differ from unity by a few percent due to EWSB (0) - (1) gauge boson mixing effects, and since we are neglecting this small effect, we take all the $\mathcal{I}=1$.

It is possible to write down an invariant top quark Yukawa coupling with either the $t_{R}=(1,1)_{2 / 3}$ or with $t_{R} \subset(1,3)_{2 / 3} \oplus(3,1)_{2 / 3}$. We refer to these possibilities as the singlet top (ST) and the triplet top (TT) models respectively, and will elaborate on both these possibilities in the following subsections. We will show the couplings relevant to the phenomenology we are interested in, controlled by the (diagonal) coupling of the new heavy fermions to the gluon (set by $g_{s}$ ), and a model dependent (off-diagonal) coupling of one heavy fermion, a "light" SM fermion and a gauge boson or the Higgs boson. The off-diagonal couplings are induced by mass mixings between the zero-mode and 1st KK mode fermions, which in turn is governed by the Yukawa couplings. We will elaborate on these couplings below.

\subsubsection{Model with $t_{R}=(1,1)_{2 / 3}$ (ST model)}

For the case of $t_{R}=(1,1)_{2 / 3}$ the kinetic-energy term is

$$
\mathcal{L}_{\mathrm{K} . \mathrm{E} .}^{t_{R}} \supset \bar{t}_{R} i \gamma^{\mu} D_{\mu} t_{R}
$$

and the top Yukawa coupling is the invariant combination $\overline{(2,2)}_{2 / 3}(2,2)_{0}(1,1)_{2 / 3}$ written as

$$
\mathcal{L}_{\text {Yuk }} \supset \lambda_{t} \operatorname{Tr}\left[\bar{Q}_{L} \Sigma\right] t_{R}+\text { h.c. . }
$$

The above $\mathcal{L}$ in eqs. (2.14) and (2.15) adds in addition to eq. (2.10) the following couplings of the component fields

$$
\begin{aligned}
& \mathcal{L}_{\mathrm{KE}} \supset g^{\prime} B_{\mu}\left[\frac{2}{3} \bar{t}_{R} \gamma^{\mu} t_{R}\right], \\
& \mathcal{L}_{\text {Yuk }} \supset \lambda_{t}\left(\bar{t}_{L} t_{R} \phi_{0}^{*}-\bar{b}_{L} t_{R} \phi^{-}+\bar{\chi} t_{R} \phi^{+}+\bar{t}^{\prime} t_{R} \phi_{0}\right)+\text { h.c. } .
\end{aligned}
$$

In the fermion sector, the mass matrix including zero-mode and (light) KK mixing but neglecting the smaller mixings to heavier KK states is

$$
\mathcal{L}_{\text {mass }} \supset\left(\bar{t}_{L} \bar{t}_{L}^{\prime}\right)\left(\begin{array}{cc}
m_{t} & 0 \\
\tilde{m} & M_{t^{\prime}}
\end{array}\right)\left(\begin{array}{c}
t_{R} \\
t^{\prime}
\end{array}\right)+\bar{b}_{L}\left(\lambda_{b} \frac{v}{\sqrt{2}}\right) b_{R}+\text { h.c. },
$$

where $m_{t}, \tilde{m}=\tilde{\lambda}_{t}(v / \sqrt{2}) f_{t_{R}}^{(n)}(\pi R) f_{t_{L}, t_{L}^{\prime}}^{(m)}(\pi R) e^{k \pi R} /(k \pi R), \tilde{\lambda}_{t} \equiv k \lambda_{t}$ is the dimensionless $5 D$ Yukawa coupling, and we have not shown mixing terms in the b-quark sector since in this model the new heavy charge $-1 / 3$ vectorlike fermions could only arise as the partners of the $b_{R}$ but we ignore them since they are very heavy. The above mass matrix is diagonalized by

$$
\left(\begin{array}{c}
t_{L} \\
t_{L}^{\prime}
\end{array}\right)=\left(\begin{array}{cc}
c_{L} & -s_{L} \\
s_{L} & c_{L}
\end{array}\right)\left(\begin{array}{l}
t_{1 L} \\
t_{2 L}
\end{array}\right) ; \quad\left(\begin{array}{c}
t_{R} \\
t_{R}^{\prime}
\end{array}\right)=\left(\begin{array}{cc}
c_{R} & -s_{R} \\
s_{R} & c_{R}
\end{array}\right)\left(\begin{array}{l}
t_{1 R} \\
t_{2 R}
\end{array}\right),
$$


where $\left\{t_{1}, t_{2}\right\}$ are the mass eigenstates (ignoring mixings to higher KK states), with the mixing angles given by

$$
\tan \left(2 \theta_{L}\right)=\frac{-2 m_{t} \tilde{m}}{\left(M_{t}^{\prime 2}-m_{t}^{2}+\tilde{m}^{2}\right)} ; \quad \tan \left(2 \theta_{R}\right)=\frac{-2 \tilde{m} M_{t}^{\prime}}{\left(M_{t}^{\prime 2}-m_{t}^{2}-\tilde{m}^{2}\right)} .
$$

The mass eigenvalues $m_{1,2}$ are given by

$$
m_{1,2}^{2}=\frac{M_{t}^{\prime 2}}{2}\left[\left(1+x_{t}^{2}+\tilde{x}^{2}\right) \mp \sqrt{\left(1+x_{t}^{2}+\tilde{x}^{2}\right)^{2}-4 x_{t}^{2}}\right],
$$

where $x_{t} \equiv m_{t} / M_{t}^{\prime}$ and $\tilde{x} \equiv \tilde{m} / M_{t}^{\prime}$. In the limit of large $M_{t}^{\prime}$, i.e., $x_{t}, \tilde{x} \ll 1$, we have

$$
m_{1}=m_{t}\left[1+O\left(x^{4}\right)\right] ; \quad m_{2}=M_{t}^{\prime}\left[1+\frac{\tilde{x}^{2}}{2}+O\left(x^{4}\right)\right] .
$$

In the mass basis the final interactions we obtain are as below. The charged current interaction is

$$
\begin{aligned}
& \mathcal{L}_{C C} \supset \frac{g_{L}}{\sqrt{2}}\left(c_{L} \bar{t}_{1 L} \gamma^{\mu} b_{L}-s_{L} \bar{t}_{2 L} \gamma^{\mu} b_{L}+s_{L} \bar{\chi}_{L} \gamma^{\mu} t_{1 L}+c_{L} \bar{\chi}_{L} \gamma^{\mu} t_{2 L}\right. \\
& \left.+s_{R} \bar{\chi}_{R} t_{1 R}+c_{R} \bar{\chi}_{R} t_{2 R}\right) W_{L \mu}^{+}+\text {h.c. . }
\end{aligned}
$$

The neutral current interaction is

$$
\begin{aligned}
\mathcal{L}_{N C} \supset & e\left[\bar{\chi} \gamma^{\mu}\left(\frac{5}{3}\right) \chi+\bar{t}_{1} \gamma^{\mu}\left(\frac{2}{3}\right) t_{1}+\bar{t}_{2} \gamma^{\mu}\left(\frac{2}{3}\right) t_{2}+\bar{b} \gamma^{\mu}\left(-\frac{1}{3}\right) b\right] A_{\mu} \\
& +g_{Z}\left\{\bar{t}_{1 L} \gamma^{\mu}\left[\frac{1}{2} \cos 2 \theta_{L}-\frac{2}{3} s_{W}^{2}\right] t_{1 L}+\bar{t}_{2 L} \gamma^{\mu}\left[-\frac{1}{2} \cos 2 \theta_{L}-\frac{2}{3} s_{W}^{2}\right] t_{2 L}\right. \\
& +\left[\bar{t}_{2 L} \gamma^{\mu}\left(-\frac{1}{2} \sin 2 \theta_{L}\right) t_{1 L}+\text { h.c. }\right] \\
& +\bar{t}_{1 R} \gamma^{\mu}\left[-\frac{1}{2} s_{R}^{2}-\frac{2}{3} s_{W}^{2}\right] t_{1 R}+\bar{t}_{2 R} \gamma^{\mu}\left[-\frac{1}{2} c_{R}^{2}-\frac{2}{3} s_{W}^{2}\right] t_{2 R} \\
& +\left[\bar{t}_{2 R} \gamma^{\mu}\left(-\frac{1}{2} s_{R} c_{R}\right) t_{1 R}+\text { h.c. }\right] \\
& \left.+\bar{b}_{L} \gamma^{\mu}\left[-\frac{1}{2}-s_{W}^{2}\left(-\frac{1}{3}\right)\right] b_{L}+\bar{\chi} \gamma^{\mu}\left[\frac{1}{2}-s_{W}^{2}\left(\frac{5}{3}\right)\right] \chi\right\} Z_{\mu}
\end{aligned}
$$

where $g_{Z} \equiv \sqrt{g_{L}^{2}+g^{\prime 2}}$. The $\chi$ interactions above include both the $L$ and $R$ chiralities. The Higgs interactions are got by replacing $v \rightarrow h$ in eq. (2.18), after which going to the mass basis using eq. (2.19) we get

$$
\begin{aligned}
\mathcal{L}_{h} & \supset \frac{\tilde{\lambda}_{t}}{\sqrt{2}} h\left[\left(c_{L} f_{t_{L}}+s_{L} f_{t_{L}^{\prime}}\right) \bar{t}_{1 L}+\left(c_{L} f_{t_{L}^{\prime}}-s_{L} f_{t_{L}}\right) \bar{t}_{2 L}\right]\left(c_{R} t_{1 R}-s_{R} t_{2 R}\right) f_{t_{R}} \frac{e^{k \pi R}}{k \pi R}+\text { h.c. }, \\
& =h\left[\left(c_{L} \frac{m_{t}}{v}+s_{L} \frac{\tilde{m}}{v}\right) \bar{t}_{1 L}+\left(c_{L} \frac{\tilde{m}}{v}-s_{L} \frac{m_{t}}{v}\right) \bar{t}_{2 L}\right]\left(c_{R} t_{1 R}-s_{R} t_{2 R}\right)+\text { h.c. },
\end{aligned}
$$

where the wavefunctions are evaluated at $\pi R$, i.e., $f_{t_{L}, t_{L}^{\prime}}(\pi R)$ is implied in the first line above, and in the second line above we have written the Higgs couplings in terms of $m_{t}, \tilde{m}$ defined below eq. (2.18). 


\subsubsection{Model with $t_{R} \subset(1,3)_{2 / 3} \oplus(3,1)_{2 / 3}($ TT model $)$}

Here we pursue another option detailed in ref. [63] in which the $t_{R}$ can be embedded into a $(1,3)_{2 / 3}$ representation, and as explained there, due to the required $P_{L R}$ invariance, a $(3,1)_{2 / 3}$ must also be added. Thus, the multiplet containing the $t_{R}$ is

$$
\psi_{t_{R}}=\psi_{t_{R}}^{\prime} \oplus \psi_{t_{R}}^{\prime \prime}=\left(\begin{array}{cc}
t_{R} / \sqrt{2} & \chi^{\prime} \\
b^{\prime} & -t_{R} / \sqrt{2}
\end{array}\right) \oplus\left(\begin{array}{cc}
t^{\prime \prime} / \sqrt{2} & \chi^{\prime \prime} \\
b^{\prime \prime} & -t^{\prime \prime} / \sqrt{2}
\end{array}\right),
$$

where $\psi_{t_{R}}^{\prime}=(1,3)_{2 / 3}$ and $\psi_{t_{R}}^{\prime \prime}=(3,1)_{2 / 3}$. The top Yukawa coupling is obtained from

$$
\mathcal{L}_{\text {Yuk }}^{t_{R}} \supset-\sqrt{2} \lambda_{t}^{\prime} \operatorname{Tr}\left[\bar{Q}_{L} \Sigma \psi_{t_{R}}^{\prime}\right]-\sqrt{2} \lambda_{t}^{\prime \prime} \operatorname{Tr}\left[\bar{Q}_{L} \psi_{t_{R}}^{\prime \prime} \Sigma\right]+\text { h.c. },
$$

and $P_{L R}$ invariance requires $\lambda_{t}^{\prime}=\lambda_{t}^{\prime \prime}$ (which we will just denote as $\lambda_{t}$ henceforth), and also $c_{\psi_{t_{R}}^{\prime}}^{\prime}=c_{\psi_{t_{R}}^{\prime \prime}}$.

After EWSB due to $\left\langle\phi_{0}\right\rangle=v / \sqrt{2}$, with the restrictions mentioned in the previous paragraph, the mass matrix is

$$
\begin{aligned}
& \mathcal{L}_{\text {mass }} \supset-\left(\begin{array}{lll}
\bar{b}_{L} & \bar{b}_{L}^{\prime} & \bar{b}_{L}^{\prime \prime}
\end{array}\right)\left(\begin{array}{ccc}
0 & \sqrt{2} m_{b b^{\prime}} & \sqrt{2} m_{b b^{\prime \prime}} \\
0 & M_{b^{\prime}} & 0 \\
0 & 0 & M_{b^{\prime \prime}}
\end{array}\right)\left(\begin{array}{c}
b_{R} \\
b_{R}^{\prime} \\
b_{R}^{\prime \prime}
\end{array}\right) \\
&-\left(\begin{array}{lll}
\bar{t}_{L} & \bar{t}_{L}^{\prime} & \bar{t}_{L}^{\prime \prime}
\end{array}\right)\left(\begin{array}{ccc}
m_{t t} & 0 & m_{t t^{\prime \prime}} \\
-m_{t^{\prime} t} & M_{t^{\prime}} & -m_{t^{\prime} t^{\prime \prime}} \\
0 & -m_{t^{\prime} t^{\prime \prime}} & M_{t^{\prime \prime}}
\end{array}\right)\left(\begin{array}{c}
t_{R} \\
t_{R}^{\prime} \\
t_{R}^{\prime \prime}
\end{array}\right) \\
&-\left(\begin{array}{llll}
\bar{\chi}_{L} & \bar{\chi}_{L}^{\prime} & \bar{\chi}_{L}^{\prime \prime}
\end{array}\right)\left(\begin{array}{ccc}
M_{\chi} & \sqrt{2} m_{\chi \chi^{\prime}} & \sqrt{2} m_{\chi \chi^{\prime \prime}} \\
\sqrt{2} m_{\chi \chi^{\prime}} & M_{\chi^{\prime}} & 0 \\
\sqrt{2} m_{\chi \chi^{\prime \prime}} & 0 & M_{\chi^{\prime \prime}}
\end{array}\right)\left(\begin{array}{c}
\chi_{R} \\
\chi_{R}^{\prime} \\
\chi_{R}^{\prime \prime}
\end{array}\right)+\text { h.c. },
\end{aligned}
$$

where the $M_{i}$ are the vectorlike masses, and the EWSB generated masses $m_{i j}$ are given by

$$
m_{i j}=\tilde{\lambda}_{t} \frac{v}{\sqrt{2}} \frac{1}{k \pi R} f_{\psi_{L}^{i}}^{(n)}(\pi R) f_{\psi_{R}^{j}}^{(m)}(\pi R) e^{k \pi R}
$$

$\tilde{\lambda}_{t} \equiv k \lambda_{t}$ is the dimensionless $5 D$ Yukawa coupling.

We will work out next the couplings in the mass basis. We write $\psi^{\alpha} \equiv\left(\psi \psi^{\prime} \psi^{\prime \prime}\right)^{T}$ and the mass eignestates as $\psi^{i} \equiv\left(\psi_{1} \psi_{2} \psi_{3}\right)^{T}$ for each of the $\psi=\{b, t, \chi\}$ sectors (here $\psi^{\prime}$ for the $t$-sector is really what we have called $t^{\prime}$ ). We perform a bi-orthogonal rotation (we take the masses to be real for simplicity) $\psi_{L}^{\alpha}=R_{\psi L}^{\alpha i} \psi_{L}^{i}$ and $\psi_{R}^{\alpha}=R_{\psi R}^{\alpha i} \psi_{R}^{i}$ to diagonalize each of the mass matrices in eq. (2.28).

The $Z$ couplings for the $\psi=\{b, t, \chi\}$-sectors in unitary gauge in the mass basis are

$$
\mathcal{L} \supset g_{Z} \bar{\psi}_{L, R}^{i}\left[R_{\psi_{L, R}}^{\alpha i^{*}}\left(q_{\psi_{L, R}^{\alpha}}^{3 L}-Q_{\psi} s_{W}^{2}\right) R_{\psi_{L, R}}^{\alpha j}\right] \gamma^{\mu} \mathcal{I}_{\psi \psi Z} \psi_{L, R}^{j} Z_{\mu}
$$

where the $q^{3 L}$ are the $W_{L}^{3}$ charges and $Q_{\psi}$ are EM charges as given below and we ignore differences in the overlap integrals and take $\mathcal{I}=1$. The $W_{L}^{3}$ charges are $q_{b_{L}^{\alpha}}^{3 L}=\{-1 / 2,0,-1\}$, 
$q_{b_{R}^{\alpha}}^{3 L}=\{0,0,-1\}, q_{t_{L}^{\alpha}}^{3 L}=\{1 / 2,-1 / 2,0\}, q_{t_{R}^{\alpha}}^{3 L}=\{0,-1 / 2,0\}, q_{\chi_{L}^{\alpha}}^{3 L}=\{1 / 2,0,1\}, q_{\chi_{R}^{\alpha}}^{3 L}=$ $\{1 / 2,0,1\}$. The EM chages are $Q_{b}=-1 / 3, Q_{t}=2 / 3$ and $Q_{\chi}=5 / 3$.

The Higgs couplings in the mass basis are

$$
\mathcal{L} \supset-\bar{\psi}_{L}^{i} R_{\psi_{L}}^{\alpha i^{*}} \frac{m^{\alpha \beta}}{v} R_{\psi_{R}}^{\beta j} \psi_{R}^{j} h+\text { h.c. },
$$

where $m^{\alpha \beta}$ are the off-diagonal EWSB induced masses in eq. (2.29).

The charged current $W^{ \pm}$interactions, in addition to those in eq. (2.13), are

$$
\mathcal{L}_{C C}^{W} \supset g_{L}\left(\bar{t}^{\prime \prime} \gamma^{\mu} b^{\prime \prime} \mathcal{I}_{t^{\prime \prime} b^{\prime \prime} W}-\bar{\chi}^{\prime \prime} \gamma^{\mu} t^{\prime \prime} \mathcal{I}_{\chi^{\prime \prime} t^{\prime \prime} W}\right) W_{L}^{+}+\text {h.c. },
$$

which in the mass basis in unitary gauge are

$$
\begin{aligned}
\mathcal{L}_{C C}^{W} \supset \frac{g_{L}}{\sqrt{2}}\left[\bar{t}_{L}^{i} R_{t_{L}}^{1 i^{*}} R_{b_{L}}^{1 j} \gamma^{\mu} b_{L}^{j}+\left(\bar{\chi}_{L}^{i} R_{\chi_{L}}^{1 i^{*}} R_{t_{L}}^{2 j} \gamma^{\mu} t_{L}^{j}\right.\right. \\
\\
\left.\left.\quad+\sqrt{2} \bar{t}_{R}^{i} R_{t_{R}}^{3 i^{*}} R_{b_{R}}^{3 j} \gamma^{\mu} b_{R}^{j}-\sqrt{2} \bar{\chi}_{R}^{i} R_{\chi_{R}}^{3 i^{*}} R_{t_{R}}^{3 j} \gamma^{\mu} t_{R}^{j}\right)+(L \leftrightarrow R)\right] W_{L}^{+}+\text {h.c. },
\end{aligned}
$$

and again we ignore differences in the overlap integrals and take $\mathcal{I}=1$.

In $\mathrm{B}$ we present analytical expressions for the mixing matrices in the $b$-quark sector in the limit of $m_{i j} / M_{\psi^{\prime}} \ll 1$, and the resulting couplings in the mass basis. We present this for illustration only and have used exact numerical diagonalization in all our results.

One way to generate the bottom-quark mass is to have a Yukawa coupling that respects the custodial symmetry. With $Q_{L}=(2,2)_{2 / 3}$, the $b_{R}$ can be embedded into the representation $\psi_{b_{R}}^{\prime}=(1,3)_{2 / 3}$ and the $b$-quark Yukawa coupling obtained from, $\mathcal{L}_{\text {Yuk }}^{b_{R}} \supset$ $-\lambda_{b}^{\prime} \operatorname{Tr}\left[\bar{Q}_{L} \Sigma \psi_{b_{R}}^{\prime}\right]+$ h.c. This breaks the $P_{L R}$ symmetry but the resulting shifts are acceptable since the $c_{b_{R}}$ choice required to get the correct $b$-quark mass makes the new vectorlike fermions in the $\psi_{b_{R}}^{\prime}$ multiplet all very heavy $(>3 \mathrm{TeV})$. In our analysis we have therefore ignored the mixing effects and the signatures of these heavy fermions. Many more possibilities for $b_{R}$ representations are discussed in ref. [63].

\section{Parameters and couplings}

The vectorlike fermions can mix among themselves and with SM fermions. We take this into account and denote the mass eigenstates by a subscript, i.e., $X_{n}$ denotes the $n^{\text {th }}$ mass eigenstate of $\mathrm{X}$ type quark except for the SM quarks where we use $t$ or $t_{1}$ and $b$ or $b_{1}$ interchangeably.

We parametrize the relevant vectorlike quark couplings model-independently as

$$
\begin{aligned}
\mathcal{L}_{\chi} \supset & \kappa_{\chi_{1 L} t_{1 L} W} \bar{\chi}_{1 L} \gamma^{\mu} t_{1 L} W_{\mu}^{+}+\kappa_{\chi_{1 R} t_{1 R} W} \bar{\chi}_{1 R} \gamma^{\mu} t_{1 R} W_{\mu}^{+}+\text {h.c. } \\
\mathcal{L}_{t^{\prime}} \supset & \kappa_{t_{2 L} t_{1 R} h} \bar{t}_{2 L} t_{1 R} h+\kappa_{t_{1 L} t_{2 R} h} \bar{t}_{1 L} t_{2 R} h \\
& +\kappa_{t_{1 L} t_{2 L} Z} \bar{t}_{1 L} \gamma^{\mu} t_{2 L} Z_{\mu}+\kappa_{t_{1 R} t_{2 R} Z} \bar{t}_{1 R} \gamma^{\mu} t_{2 R} Z_{\mu} \\
& +\kappa_{t_{2 L} \chi_{1 L} W} \bar{t}_{2 L} \gamma^{\mu} \chi_{1 L} W_{\mu}+\kappa_{t_{2 R} \chi_{1 R} W} \bar{t}_{2 R} \gamma^{\mu} \chi_{1 R} W_{\mu}+\text { h.c. } \\
\mathcal{L}_{b^{\prime}} \supset & \kappa_{b_{2 L} b_{1 L} Z} \bar{b}_{2 L} \gamma^{\mu} b_{1 L} Z_{\mu}+\kappa_{b_{2 R} b_{1 R} Z} \bar{b}_{2 R} \gamma^{\mu} b_{1 R} Z_{\mu} \\
& +\kappa_{b_{2 L} b_{1 R} h} \bar{b}_{2 L} b_{1 R} h+\kappa_{b_{2 R} b_{1 L} h} \bar{b}_{2 R} b_{1 L} h+\text { h.c. }
\end{aligned}
$$

Wherever possible we show results model-independently as functions of the $\kappa$ 's defined above. 

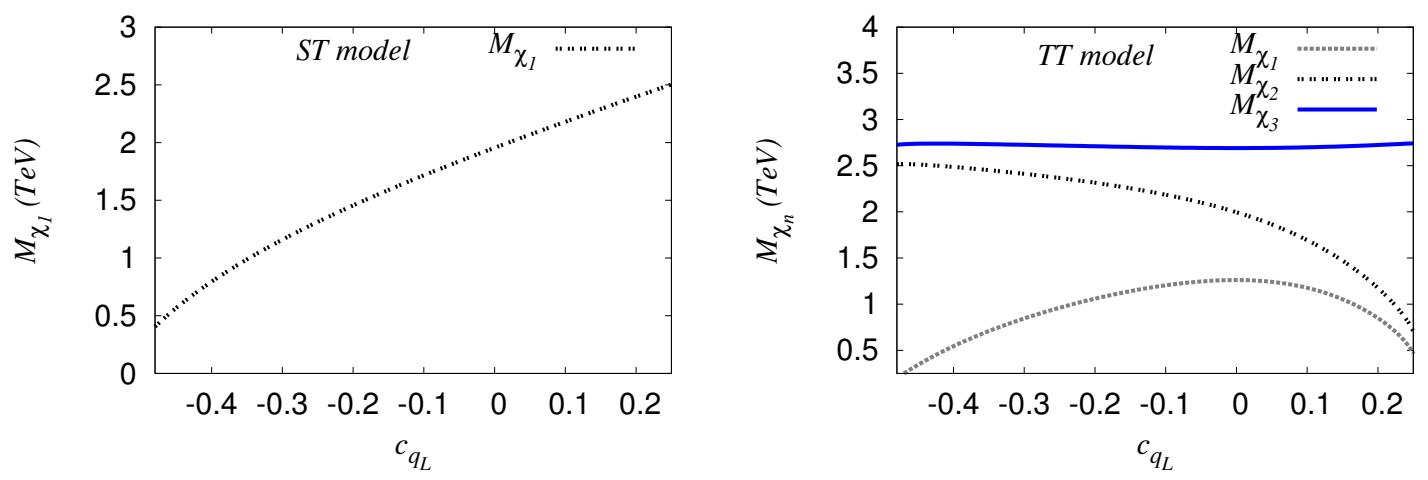

Figure 1. $M_{\chi_{1}, \chi_{2}, \chi_{3}}$ as functions of $c_{q_{L}}$ in the ST and TT models with $\tilde{\lambda}_{t}=1, \tilde{\lambda}_{b}=1$ and $M_{K K}=3 \mathrm{TeV}$.

In the following, we present the parameter choices we make for the different warped models discussed in section 2 for which we present numerical results. The analytical expressions for the fermion mass eigenvalue, the fermion profiles along the extra dimensions, and their dependence on the $c$-parameters are given in $\mathrm{A}^{2}{ }^{2}$ Using these profiles, we compute the overlap integrals and determine the couplings of the vectorlike fermions to the SM states relevant to our study. Various choices of the three relevant $c$-parameters, namely $c_{q_{L}}, c_{t_{R}}$ and $c_{b_{R}}$, are possible that reproduce the measured masses and couplings (see for e.g. refs. $[75,76]$ and references therein). Furthermore, there is freedom to choose the 5D Yukawa couplings $\tilde{\lambda}$ which we set to 1 , and $M_{K K}$ which we take to be $3 \mathrm{TeV}$. After these choices and imposing the constraint that the lightest eigenvalues in the top and bottom quark sectors correspond to the measured top mass $(172 \mathrm{GeV})$ and bottom mass $(4.2 \mathrm{GeV})$ respectively, there is one free parameter remaining which we take to be $c_{q_{L}}$. In the following, we show some representative benchmark points for the various warped models detailed in section 2 , for each of the $\chi, t^{\prime}$ and $b^{\prime}$.

\section{$3.1 \chi$ parameters and couplings}

The $\kappa$ for the warped model are as detailed in section 2. In figures 1 and 2 , we show $M_{\chi_{1}}$, $\kappa_{\chi_{1 L} t_{1 L} W}$ and $\kappa_{\chi_{1 R} t_{1 R} W}$ as functions of $c_{q_{L}}$ for the $Z b \bar{b}$ protected ST and TT models. There is no $\chi$ state in the DT model. In the TT model, after $\chi-\chi^{\prime}-\chi^{\prime \prime}$ mixing, the $\chi_{2}, \chi_{3}$ becomes much heavier than $\chi_{1}$ because the appearance of the large off-diagonal term in the $\chi$ mass matrix causes a significant split between $M_{\chi_{1}}$ and $M_{\chi_{2}, \chi_{3}}$. Therefore, for both ST and TT models, we focus only on the phenomenology of $\chi_{1}$. In the TT model $M_{\chi_{1}}$ in figure 1 shows an unusual behavior - with increasing $c_{Q_{L}}$, it first increases and then decreases. This is an effect of the diagonalization of eq. (2.28), with $c_{q_{L}} \lesssim 0$ having $M_{\chi}<M_{\chi^{\prime}}$ while $c_{q_{L}} \gtrsim 0$ has $M_{\chi}>M_{\chi^{\prime}}$, and the maximum of the eigenvalue is attained when $M_{\chi}=M_{\chi^{\prime}}$. In table 1 we explicitly display the benchmark parameters and couplings in the ST model that we use for our numerical computations when we discuss $\chi_{1}$ phenomenology. In the

\footnotetext{
${ }^{2}$ We find that after mixing the couplings relevant for our study are largely insensitive to the choice of $k \pi R$ and $\tilde{\lambda}_{b, t}$; for instance, for $M_{K K}=3 \mathrm{TeV}$, varying $k / M_{P l}$ between 0.1 and 1 changes the couplings by at most $1 \%$ and varying $\tilde{\lambda}_{b, t}$ between 1 and 2 changes couplings only about a few percent.
} 

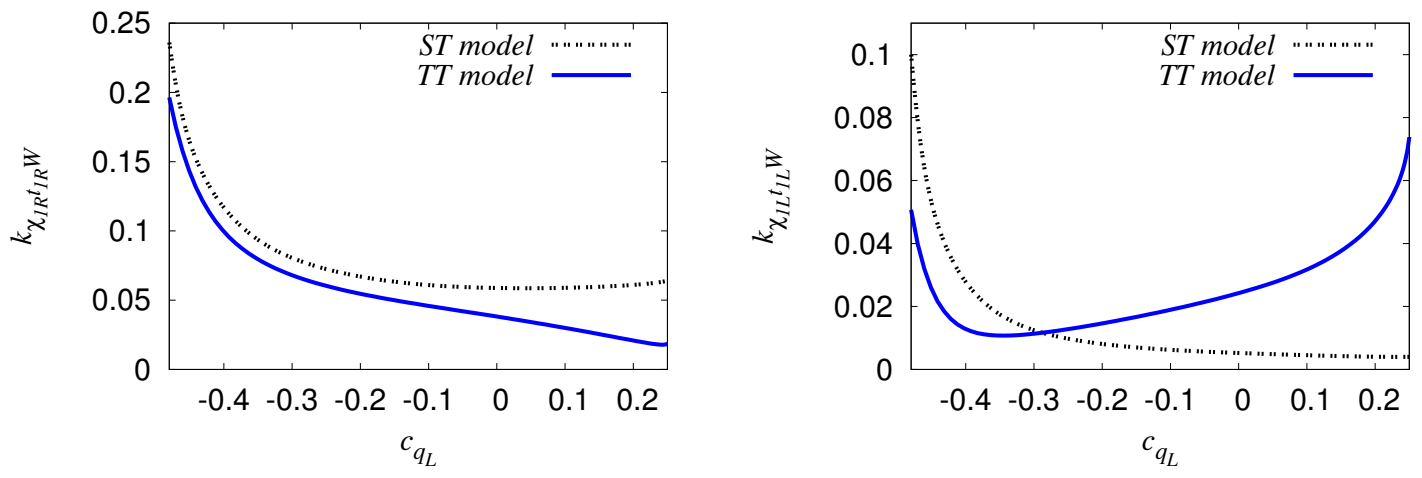

Figure 2. $\kappa_{\chi_{1} t W}$ 's as functions of $c_{q_{L}}$ in the ST and TT models with $\tilde{\lambda}_{t}=1, \tilde{\lambda}_{b}=1$ and $M_{K K}=3 \mathrm{TeV}$.

\begin{tabular}{|c|c|c|c|c|c|}
\hline $\mathcal{X}$ & $c_{q_{L}}$ & $c_{t_{R}}$ & $c_{b_{R}}$ & $\sin \theta_{L}$ & $\sin \theta_{R}$ \\
\hline $\mathcal{X}_{1}$ & -0.463 & 0.206 & 0.586 & -0.136 & -0.394 \\
$\mathcal{X}_{2}$ & -0.414 & 0.216 & 0.585 & -0.058 & -0.253 \\
$\mathcal{X}_{3}$ & -0.350 & 0.202 & 0.584 & -0.033 & -0.192 \\
$\mathcal{X}_{4}$ & -0.274 & 0.177 & 0.583 & -0.022 & -0.159 \\
$\mathcal{X}_{5}$ & -0.186 & 0.137 & 0.581 & -0.016 & -0.140 \\
$\mathcal{X}_{6}$ & -0.088 & 0.078 & 0.578 & -0.013 & -0.129 \\
\hline \hline $\mathcal{X}$ & $M_{\chi}(\mathrm{GeV})$ & $\kappa_{\chi_{1 R} t_{1 R} W}$ & $\kappa_{\chi_{1 L} t_{1 L} W}$ & $\kappa_{\chi_{1 R} t_{2 R} W}$ & $\kappa_{\chi_{1 L} t_{2 L} W}$ \\
\hline $\mathcal{X}_{1}$ & 500 & 0.182 & 0.063 & 0.424 & 0.458 \\
$\mathcal{X}_{2}$ & 750 & 0.117 & 0.027 & 0.447 & 0.461 \\
$\mathcal{X}_{3}$ & 1000 & 0.089 & 0.015 & 0.453 & 0.462 \\
$\mathcal{X}_{4}$ & 1250 & 0.074 & 0.010 & 0.456 & 0.462 \\
$\mathcal{X}_{5}$ & 1500 & 0.065 & 0.007 & 0.457 & 0.462 \\
$\mathcal{X}_{6}$ & 1750 & 0.060 & 0.006 & 0.458 & 0.462 \\
\hline
\end{tabular}

Table 1. $\chi$ benchmark parameters (parameter set denoted by $\mathcal{X}$ ) and couplings obtained using $\tilde{\lambda}_{t}=1, \tilde{\lambda}_{b}=1$ and $M_{K K}=3 \mathrm{TeV}$ in the ST model. The $c$ values for all the benchmark parameter sets reproduce correct top and bottom quark masses after mixing.

ST model, we restrict ourselves to $c_{q_{L}}<0$, i.e. with the $q_{L}$ partners peaked towards the IR brane, since otherwise the partners become very heavy and this may be out of reach at the LHC.

In the TT model we have $M_{\chi^{\prime}}=M_{\chi^{\prime \prime}}$ due to the $P_{L R}$ symmetry of the theory and we find the $\chi_{2} \chi_{1} h$ couplings (both $L$ and $R$ ) to be zero as a consequence of this. The $\chi_{2} \chi_{3} h$ coupling is also zero. Furthermore, the $P_{L R}$ symmetry also constrains $m_{\chi \chi^{\prime}}=m_{\chi \chi^{\prime \prime}}$ and as a result we find $\chi_{3} \chi_{1} Z$ (both $L$ and $R$ ) couplings to be zero. 

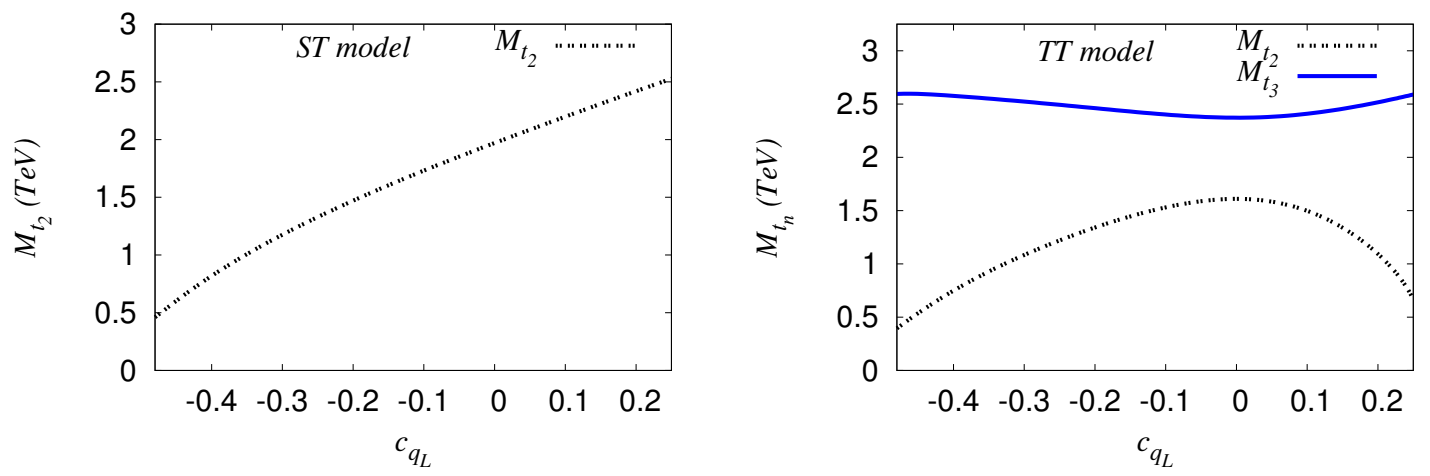

Figure 3. $M_{t_{2}, t_{3}}$ as functions of $c_{q_{L}}$ in the ST and TT models with $\tilde{\lambda}_{t}=1, \tilde{\lambda}_{b}=1$ and $M_{K K}=$ $3 \mathrm{TeV}$.

\section{$3.2 \quad t^{\prime}$ parameters and couplings}

The $\kappa$ for the warped model are as detailed in section 2. In the model with no $Z b \bar{b}$ protection (DT model), the $t^{\prime}$ is quite heavy (above $3 \mathrm{TeV}$ ) due to the choice of the $c_{b_{R}}$ required for the correct $b$-quark mass, making its LHC discovery challenging. We therefore will not discuss further the $t^{\prime}$ in the DT model, and will restrict ourselves to the $Z b \bar{b}$ protected ST and TT models. In figure 3 we show the $M_{t^{\prime}}$ as functions of $c_{q_{L}}$ in the ST and TT models. For the TT model, we note that the mass eigenvalue $M_{t_{2}}$ shows a similar behavior as $M_{\chi_{1}}$, i.e., with increasing $c_{Q_{L}}$, it first increases and then decreases. We also find for the TT model that the $t_{2-} \chi_{1}$ mass-difference is larger than $m_{W}$ which allows the $t_{2} \rightarrow \chi_{1} W$ decay mode. We show the $t_{2}$ couplings in figure 4 for the various models as functions of $c_{q_{L}}$. $\kappa_{t_{2} \chi_{1} W}$ is large since it is given by the $t^{\prime} \chi W$ or $t^{\prime \prime} \chi^{\prime \prime} W$ couplings, and is not proportional to any small off-diagonal mixing-matrix elements. In table 2 we display the benchmark parameters and couplings in the ST model that are used for our numerical computations.

\section{$3.3 \quad b^{\prime}$ parameters and couplings}

The $\kappa$ for the warped model are as detailed in section 2. As already mentioned, our convention of the Higgs coupling $\kappa$ 's appearing in eq. (2.5) differ by a factor of $\sqrt{2}$ compared to that in ref. [1]. We display $M_{b_{2}}, \kappa_{b_{2} b_{1} h}$ and $\kappa_{b_{2} b_{1} Z}$ as functions of $c_{q_{L}}$ for the DT and TT models in figures 5 and 6 . In the TT model we have $M_{b^{\prime}}=M_{b^{\prime \prime}}$ due to the $P_{L R}$ symmetry of the theory and we find that the $b_{2} b_{1} h$ couplings (both $L$ and $R$ ) to be zero as a consequence of this. The $b_{2} b_{3} h$ coupling is also zero. Furthermore, the $P_{L R}$ symmetry also constrains $m_{b b^{\prime}}=m_{b b^{\prime \prime}}$ and as a result we find $b_{3} b_{1} Z$ (both $L$ and $R$ ) couplings to be zero. These are explicitly seen in the analytical formulas shown in B in the small mixing limit. In table 3 we show the parameters for some benchmark points in the TT model. $R_{b_{L}}^{12}$ and $R_{b_{R}}^{12}$ are as defined in section 2.2.2. We have $V_{t b}=R_{t_{L}}^{11 *} R_{b_{L}}^{11}$, and for the lower $b^{\prime}$ masses this may be somewhat close to the experimental limit quoted earlier. In $\mathrm{B}$ we give the analytical expressions in the TT model in the small mixing limit for illustration, and use exact numerical diagonalization in our results. 

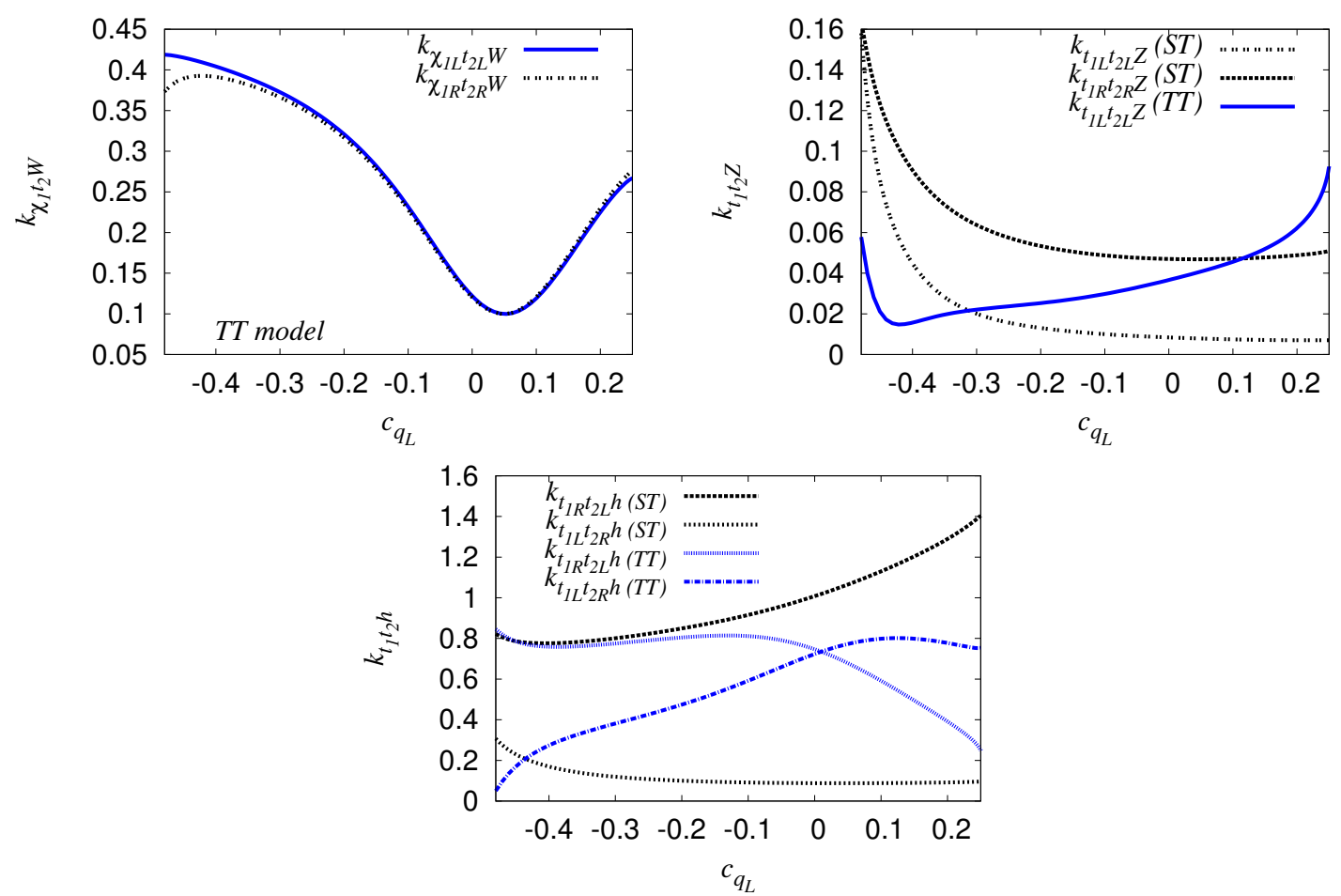

Figure 4. The $\kappa$ 's for $t_{2}$ as functions of $c_{q_{L}}$ in the ST and TT models, with $\tilde{\lambda}_{t}=1, \tilde{\lambda}_{b}=1$ and $M_{K K}=3 \mathrm{TeV}$.

\begin{tabular}{|c|c|c|c|c|c|}
\hline $\mathcal{T}$ & $c_{q_{L}}$ & $c_{t_{R}}$ & $c_{b_{R}}$ & $\sin \theta_{L}$ & $\sin \theta_{R}$ \\
\hline $\mathcal{T}_{1}$ & -0.471 & 0.196 & 0.586 & -0.167 & -0.442 \\
$\mathcal{T}_{2}$ & -0.419 & 0.216 & 0.585 & -0.062 & -0.262 \\
$\mathcal{T}_{3}$ & -0.356 & 0.204 & 0.584 & -0.034 & -0.195 \\
$\mathcal{T}_{4}$ & -0.279 & 0.179 & 0.583 & -0.022 & -0.161 \\
$\mathcal{T}_{5}$ & -0.191 & 0.140 & 0.581 & -0.016 & -0.141 \\
$\mathcal{T}_{6}$ & -0.094 & 0.082 & 0.578 & -0.013 & -0.130 \\
\hline \hline $\mathcal{T}$ & $M_{t_{2}}(\mathrm{GeV})$ & $\kappa_{t_{2 L} t_{1 R} h}$ & $\kappa_{t_{1 L} t_{2 R} h}$ & $\kappa_{t_{2 R} t_{1 R} Z}$ & $\kappa_{t_{2 L} t_{1 L} Z}$ \\
\hline $\mathcal{T}_{1}$ & 500 & 0.806 & 0.277 & 0.148 & 0.123 \\
$\mathcal{T}_{2}$ & 750 & 0.769 & 0.176 & 0.094 & 0.046 \\
$\mathcal{T}_{3}$ & 1000 & 0.778 & 0.134 & 0.071 & 0.026 \\
$\mathcal{T}_{4}$ & 1250 & 0.807 & 0.111 & 0.059 & 0.017 \\
$\mathcal{T}_{5}$ & 1500 & 0.851 & 0.098 & 0.052 & 0.012 \\
$\mathcal{T}_{6}$ & 1750 & 0.915 & 0.090 & 0.048 & 0.010 \\
\hline
\end{tabular}

Table 2. $t_{2}$ benchmark parameters (parameter set denoted by $\mathcal{T}$ ) and couplings obtained using $\tilde{\lambda}_{t}=1, \tilde{\lambda}_{b}=1$ and $M_{K K}=3 \mathrm{TeV}$ in the ST model. The $c$ values for all the benchmark parameter sets reproduce correct top and bottom quark masses after mixing. 

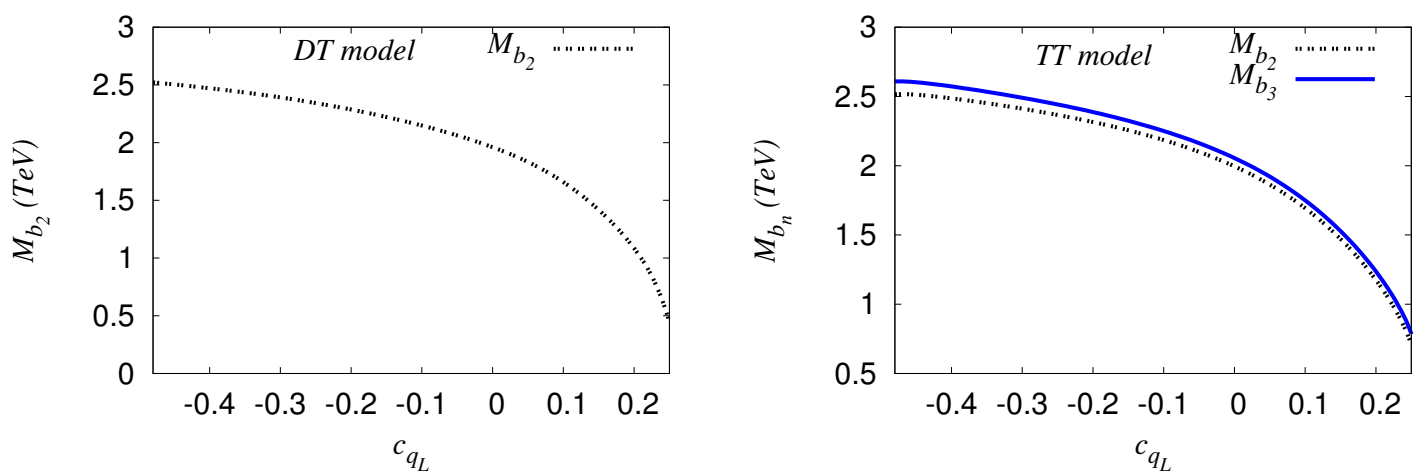

Figure 5. $M_{b_{2}, b_{3}}$ as functions of $c_{q_{L}}$ in the DT and TT models, with $\tilde{\lambda}_{t}=1, \tilde{\lambda}_{b}=1$ and $M_{K K}=3 \mathrm{TeV}$.
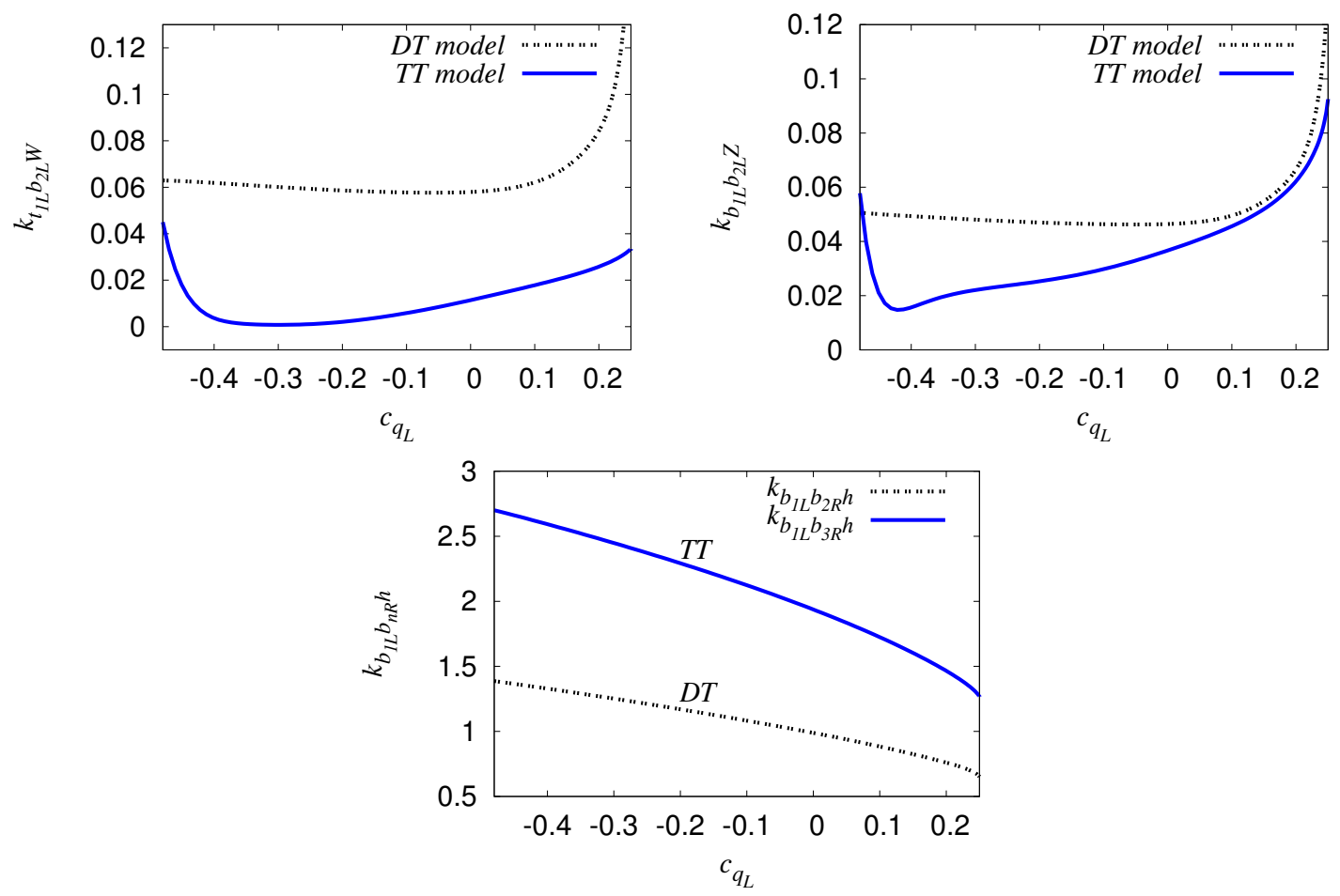

Figure 6. The $\kappa$ 's for $b_{2}$ as functions of $c_{q_{L}}$ in the DT and TT models, with $\tilde{\lambda}_{t}=1, \tilde{\lambda}_{b}=1$ and $M_{K K}=3 \mathrm{TeV}$. 


\begin{tabular}{|c|c|c|c|c|c|}
\hline $\mathcal{B}$ & $c_{q_{L}}$ & $c_{t_{R}}$ & $c_{b_{R}}$ & $R_{b_{L}}^{12}$ & $R_{b_{R}}^{12}$ \\
\hline $\mathcal{B}_{1}$ & 0.259 & -0.464 & 0.562 & -0.400 & -0.0034 \\
$\mathcal{B}_{2}$ & 0.247 & -0.414 & 0.566 & -0.299 & -0.0017 \\
$\mathcal{B}_{3}$ & 0.226 & -0.350 & 0.569 & -0.242 & -0.0010 \\
$\mathcal{B}_{4}$ & 0.197 & -0.274 & 0.571 & -0.207 & -0.0007 \\
$\mathcal{B}_{5}$ & 0.156 & -0.186 & 0.574 & -0.186 & -0.0005 \\
$\mathcal{B}_{6}$ & 0.098 & -0.088 & 0.577 & -0.173 & -0.0004 \\
\hline \hline $\mathcal{B}$ & $M_{b_{2}}(\mathrm{GeV})$ & $\kappa_{b_{2 L} t_{1 L} W}$ & $\kappa_{b_{2 L} b_{1 L} Z}$ & $\kappa_{b_{2 L} t_{2 L} W}$ & $\kappa_{b_{2 R} t_{2 R} W}$ \\
\hline $\mathcal{B}_{1}$ & 500 & 0.118 & 0.210 & 0.300 & 0.322 \\
$\mathcal{B}_{2}$ & 750 & 0.077 & 0.158 & 0.311 & 0.321 \\
$\mathcal{B}_{3}$ & 1000 & 0.060 & 0.128 & 0.313 & 0.319 \\
$\mathcal{B}_{4}$ & 1250 & 0.050 & 0.109 & 0.311 & 0.315 \\
$\mathcal{B}_{5}$ & 1500 & 0.044 & 0.098 & 0.303 & 0.306 \\
$\mathcal{B}_{6}$ & 1750 & 0.041 & 0.091 & 0.283 & 0.286 \\
\hline
\end{tabular}

Table 3. $b_{2}$ benchmark parameters (parameter set denoted by $\mathcal{B}$ ) and couplings obtained using $\tilde{\lambda}_{t}=1, \tilde{\lambda}_{b}=1$ and $M_{K K}=3 \mathrm{TeV}$ in the TT model. The $c$ values for all the benchmark parameter sets reproduce correct top and bottom quark masses after mixing.

\section{Decay width and branching ratio}

Here, we present the decay width and branching ratios (BRs) of vectorlike quarks. As concrete examples we take the different models detailed in section 2, namely the DT, ST and TT models.

The analytical expressions for the vectorlike fermion partial decay widths are ${ }^{3}$

$$
\begin{aligned}
\Gamma_{q_{2} \rightarrow q_{1} V}= & \frac{1}{32 \pi} \frac{M_{q_{2}}^{3}}{M_{V}^{2}}\left[\left(\kappa_{L}^{2}+\kappa_{R}^{2}\right)\left\{\left(1-x_{q_{1}}^{2}\right)^{2}+x_{V}^{2}\left(1+x_{q_{1}}^{2}\right)-2 x_{V}^{4}\right\}\right. \\
& \left.-12 \kappa_{L} \kappa_{R} x_{q_{1}} x_{V}^{2}\right] \times\left(1+x_{q_{1}}^{4}+x_{V}^{4}-2 x_{q_{1}}^{2}-2 x_{V}^{2}-2 x_{q_{1}}^{2} x_{V}^{2}\right)^{\frac{1}{2}} \\
\Gamma_{q_{2} \rightarrow q_{1} h}= & \frac{1}{32 \pi} M_{q_{2}}\left[\left(\kappa_{L}^{2}+\kappa_{R}^{2}\right)\left\{\left(1-x_{q_{1}}^{2}-x_{h}^{2}\right)^{2}\right\}+4 \kappa_{L} \kappa_{R} x_{q_{1}}\right] \\
& \times\left(1+x_{q_{1}}^{4}+x_{h}^{4}-2 x_{q_{1}}^{2}-2 x_{h}^{2}-2 x_{q_{1}}^{2} x_{h}^{2}\right)^{\frac{1}{2}},
\end{aligned}
$$

where the $\kappa_{L, R}$ are the couplings parametrized as in section 3 , and $x_{q_{1}} \equiv M_{q_{1}} / M_{q_{2}}, x_{V} \equiv$ $M_{V} / M_{q_{2}}$ and $x_{h} \equiv M_{h} / M_{q_{2}}$. We can obtain the total width and BRs in any model containing vectorlike fermions using the above equations. Next, we present some results for the warped models.

\footnotetext{
${ }^{3}$ The eqs. (3)-(5) of ref. [1] are special cases of these formulas. We point out a minor error in eq. (5) of ref. [1] introduced by an ambiguity in specifying a number multiplying the $4 \times 4$ identity in the program FORM. The decay width $\Gamma\left(b^{\prime} \rightarrow b h\right)$ shown in eq. (5) of ref. [1] should read as shown here in eq. (4.2). Since the error is in terms suppressed as $m_{b} / M_{b^{\prime}}$, which is small, the error does not change any of the results of that paper.
} 

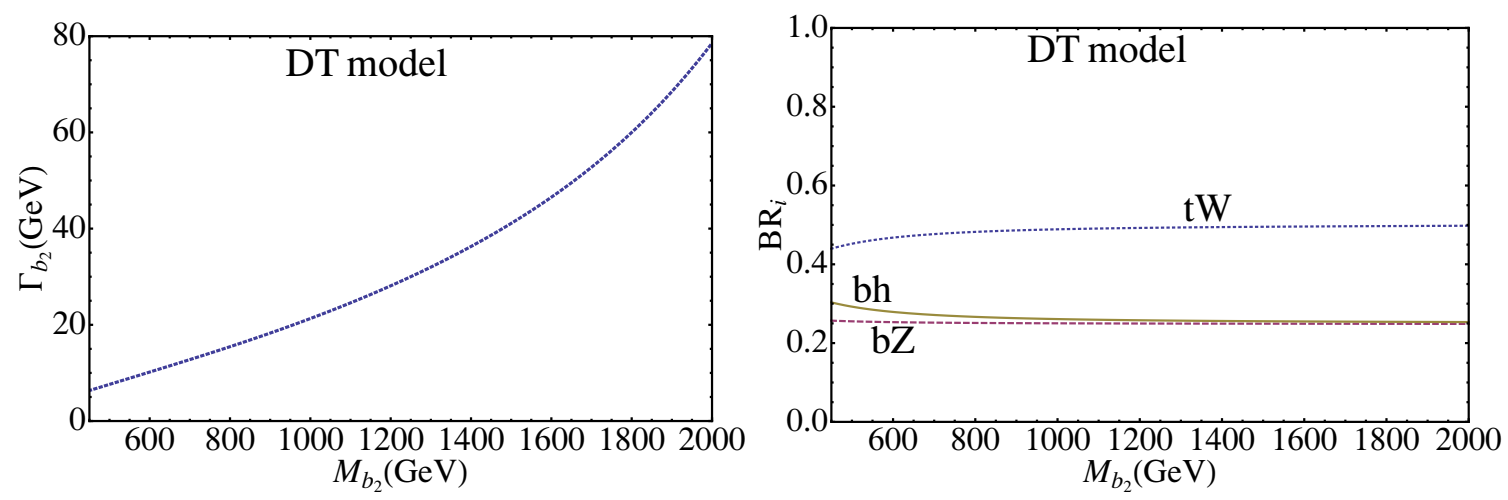

Figure 7. Total decay width and branching ratios of $b_{2}$ as functions of $M_{b_{2}}$ in the model without $Z b \bar{b}$ protection for the DT model.

\begin{tabular}{|c|c|c|c|c|c|c|c|}
\hline$M_{b_{2}}(\mathrm{GeV})$ & 500 & 750 & 1000 & 1250 & 1500 & 1750 & 2000 \\
\hline$b_{2} \rightarrow t_{1} W$ & 0.452 & 0.480 & 0.489 & 0.493 & 0.495 & 0.497 & 0.498 \\
$b_{2} \rightarrow b_{1} Z$ & 0.292 & 0.269 & 0.261 & 0.257 & 0.255 & 0.254 & 0.253 \\
$b_{2} \rightarrow b_{1} h$ & 0.255 & 0.251 & 0.250 & 0.250 & 0.249 & 0.249 & 0.249 \\
\hline
\end{tabular}

Table 4. $b_{2}$ branching ratios for the warped-space DT model.

In the warped model without custodial protection of the $Z b \bar{b}$ coupling, presented in section 2.1 (DT model), the new vectorlike fermions are the $b^{\prime}$ and $t^{\prime}$. We first focus on the on the $b^{\prime}$ here, and will present the $t^{\prime}$ decay width and BRs in the context of the ST and TT models later. In figure 7 we show the total decay width (left) and BRs (right) as functions of $M_{b_{2}}$ for the $b^{\prime}$ in the DT model. The total width is a few percent of the mass. Its roughly linear dependence on $M_{b_{2}}$ can be understood by noting from eqs. (4.1) and (4.2) that (in the large $M_{b_{2}}$ limit) $s_{\theta}^{L} \propto 1 / M_{b_{2}}, c_{\theta}^{L} \approx 1$, leaving a $\Gamma_{i} \sim M_{b_{2}}$ behavior for all the partial widths. All three modes have comparable branching ratios. For the $t W$ channel, for $M_{b_{2}}$ not too much bigger than $m_{t}$, the phase space suppression due to the large top mass is significant, but is overcome for large $M_{b_{2}}$. The $b Z$ and $b h$ BR curves are quite similar, particularly for large $M_{b_{2}}$, since, neglecting the (small) $x_{b}$, the $b_{2} b_{1} Z$ and $b_{2} b_{1} h$ couplings are proportional to $g_{Z} c_{\theta}^{L} s_{\theta}^{L}$ and $c_{\theta}^{L} \lambda_{Q_{L} b_{R}^{\prime}}$ respectively. Since $s_{\theta}^{L} \propto \lambda_{Q_{L} b_{R}^{\prime}}$ and in the $b Z$ partial width the factor of $g_{Z}^{2}$ cancels against the $1 / m_{Z}^{2}$, the two BRs end up being equal as can be shown using eq. (4.1). In table 4 we give the $b_{2}$ branching ratio for each of the three channels as a function of its mass in the DT model. In figure 8 we show the total decay width and BRs of the $b_{2}$ and $b_{3}$ for the TT model. An additional decay mode $b_{2} \rightarrow t_{2} W$ opens up at large $M_{b_{2}}$. Since this BR is not too big for the masses of interest, we do not consider this mode further.

In figure 9 we present the $t_{2}$ decay width and branching ratio for the ST model, and in figure 10 for the TT model. We notice that the $t_{2} \rightarrow b W$ decay width becomes small at large $m_{2}$. The reason for this is that there is no $T b \phi^{+}$coupling in eq. (2.17) and it will be generated after mixing as a $t_{2} b \phi^{+}$term. This is of $\mathcal{O}(\tilde{x})$ and is negligible in the large $m_{2}$ limit. In the TT model, the additional decay mode $t_{2} \rightarrow \chi_{1} W$ is present, and ends 

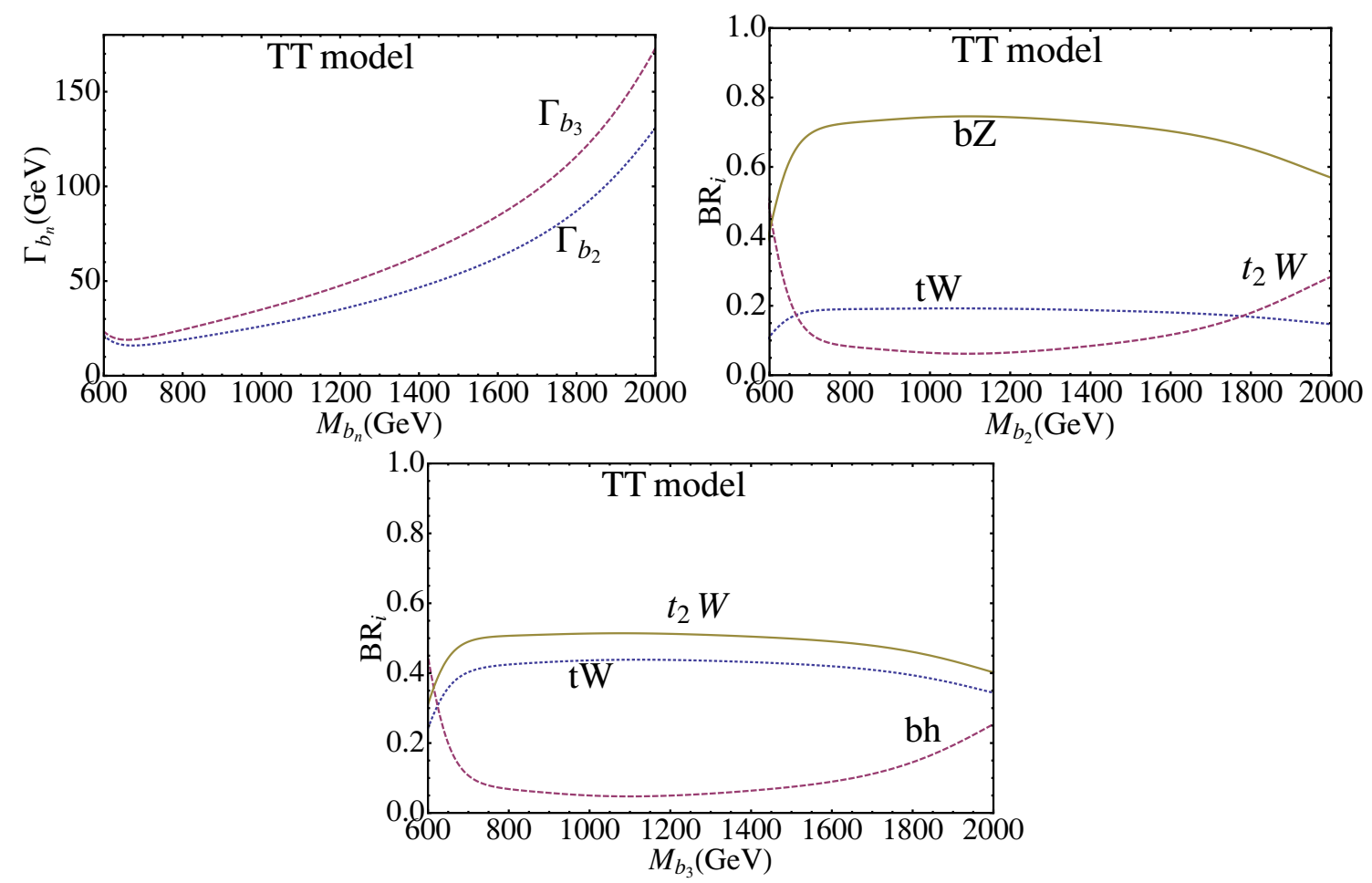

Figure 8. Total decay width (left) and branching ratios of $b_{2}$ (center) and $b_{3}$ (right) as functions of their masses in the model with $Z b \bar{b}$ protection for the TT model.
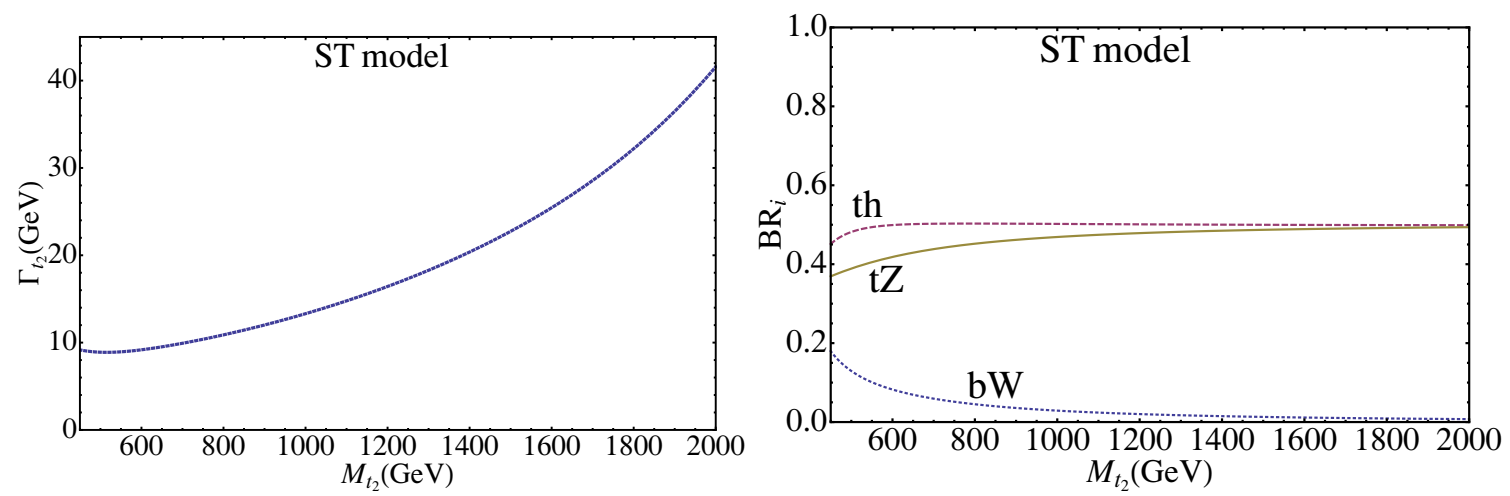

Figure 9. Total decay width and branching ratios of $t_{2}$ as functions of $M_{t_{2}}$ in the model with $Z b \bar{b}$ protection for the ST model.

up being the dominant decay mode. The reason for this is the large coupling relevant here for the reason mentioned in section 3.2. For the TT model, we show different plots for $c_{q_{L}}<0$ and $c_{q_{L}}>0$, because $M_{t_{2}}$ is two-fold degenerate for different $c_{q_{L}}$ as can be seeen from figure 3. For $c_{q_{L}}<0$ the $t_{2} \rightarrow t Z \mathrm{BR}$ is quite small while for $c_{q_{L}}>0$ it increases to about 0.2 .

In figure 11 we show the $\chi_{1}$ total decay width for the ST and TT models. The $\chi_{1} \mathrm{BR}$ is $100 \%$ into the $t W$ mode as this is the only channel accessible. For the TT model, we show different plots for $c_{q_{L}}<0$ and $c_{q_{L}}>0$ as, like $M_{t_{2}}, M_{\chi_{1}}$ also shows degeneracy as a 

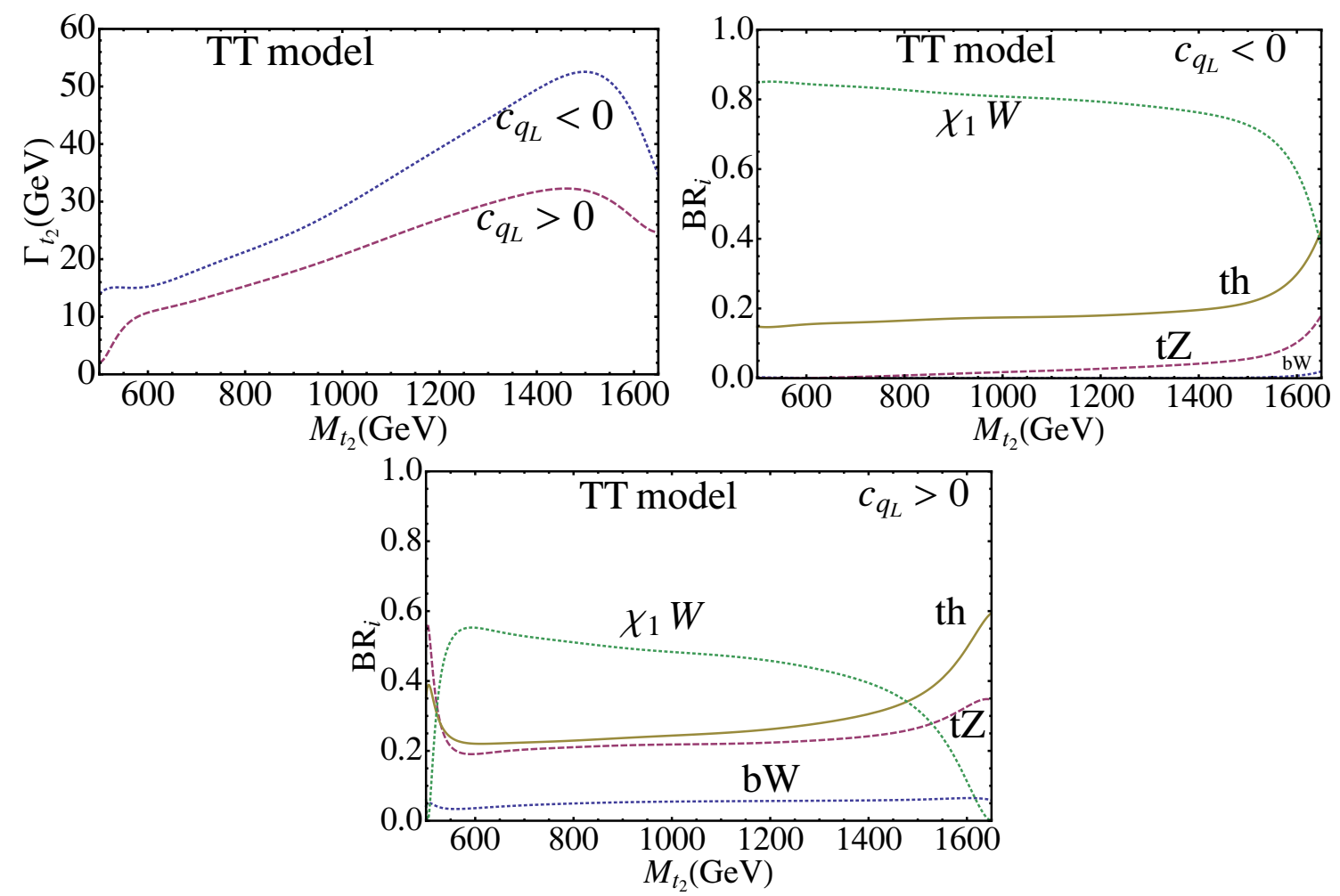

Figure 10. Total decay width and branching ratios of $t_{2}$ as functions of $M_{t_{2}}$ in the model with $Z b \bar{b}$ protection for the TT model. For the TT model, we show different plots for $c_{q_{L}}<0$ and $c_{q_{L}}>0$ becuase $M_{t_{2}}$ is two-fold degenerate for different $c_{q_{L}}$ as can be seen from figure 3 .
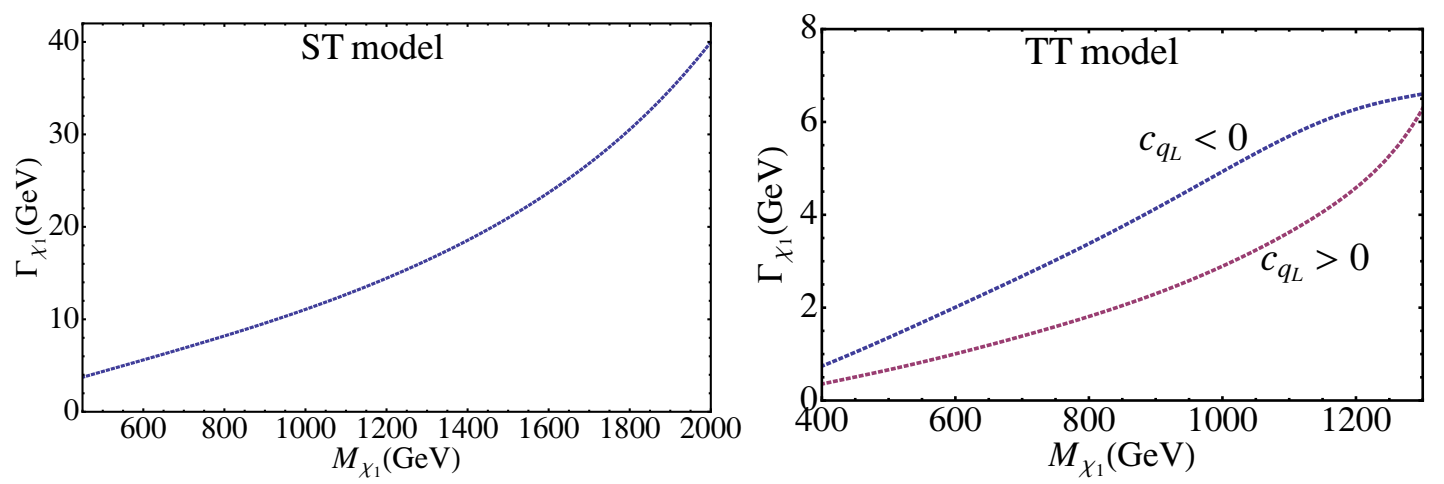

Figure 11. Total decay widths of $\chi_{1}$ as functions of $M_{\chi_{1}}$ in the ST and TT models. For the TT model, we show different plots for $c_{q_{L}}<0$ and $c_{q_{L}}>0$ because $M_{\chi_{1}}$ is two-fold degenerate for different $c_{q_{L}}$ as can be seen from figure 1 .

function of $c_{q_{L}}$ (see figure 1). In the TT model, the additional decay mode $\chi_{2} \rightarrow \chi_{1} Z$ is present, and ends up being the dominant decay mode (with BR about 0.8). The reason for this is the large coupling. Interestingly, $\chi_{2}$ has many more decay modes, namely $t W$ (with BR of about 0.2 ), $\chi_{1} Z, \chi_{1} h$, and $t_{2} W$, but we do not consider the $\chi_{2}$ as we expect its production c.s. to be smaller owing to its larger mass. 


\section{LHC signatures}

In this section we study the LHC signatures of the $\chi$ (EM charge $5 / 3$ ), $t^{\prime}$ (charge $2 / 3$ ) and $b^{\prime}$ (charge $-1 / 3$ ) vectorlike quarks. We present many of our results model-independently and also show specific signatures and the reach for the different warped models detailed in section 2, namely, the model without custodial protection of $Z b \bar{b}$ (DT model), and the two cases with custodial protection, singlet $t_{R}$ (ST model) and triplet $t_{R}$ (TT model). The warped model parameter choices we use for our numerical studies are given in section 3 .

Generally, at the LHC, the dominant production channel of these quarks is their pair production. However in this paper, in addition to the pair productions, we also look into some of their important single production channels. The single production channels can give useful information about model dependent weak coupling parameters and thus, help us to identify the underlying model at colliders. Single production can also have less complications from combinatorics compared to pair-production. Moreover, in general, depending on the coupling, some single production channel can even be the dominant production channel if the vectorlike quark is too heavy due to the phase-space suppression in pair-production. For instance, for electroweak size couplings, the single production starts to dominate for masses roughly above $700 \mathrm{GeV}$.

Due to mixing of the SM top and bottom quarks with the $t^{\prime}$ and $b^{\prime}$ respectively, $V_{t b}$ can be shifted. The current measured value of $\left|V_{t b}\right|$ from the direct measurement of the single top production cross section at the Tevatron with $\sqrt{s}=1.96 \mathrm{TeV}$ is $\left|V_{t b}\right|=0.88 \pm 0.07$ with a limit [77] of $\left|V_{t b}\right|>0.77$ at the $95 \%$ C.L. assuming a top quark mass $m_{t}=170 \mathrm{GeV}$. While presenting the results for the warped models, the parameters we use for numerical computations satisfy the above $\left|V_{t b}\right|$ constraint.

For each of the $\chi, t^{\prime}$, and $b^{\prime}$ we identify promising pair and single production channels, compute the signal cross-section and dominant SM backgrounds, and compute the luminosity required $\left(\mathcal{L}_{5}\right)$ for $5 \sigma$ significance, i.e. $S / \sqrt{B}=5$, and additionally $\left(\mathcal{L}_{10}\right)$ for obtaining 10 signal events. We take the larger of $\mathcal{L}_{5}$ and $\mathcal{L}_{10}$ as the luminosity for discovery.

We have implemented the warped model Lagrangian in FeynRules version 1.6.0 [78] and generated the model files for the Monte-Carlo event-generator MadGraph5 [79], using which we obtain the signal cross-sections. We use CTEQ6L1 Parton Distribution Functions (PDFs) [80]. We perform a patron-level study, and do not include hadronization and detector resolution effects in this first level of study.

\section{$5.1 \chi$ LHC signatures}

We assume that the only decay is $\chi \rightarrow t W$, which is the case in many BSM scenarios. We parametrize the $\chi$ couplings model-independently as shown in eq. (3.1). At the LHC, we consider the $\chi t W$ production process as we find this to be the dominant $\chi$ production channel. As shown in figure 12, this includes (i) the double resonant (DR) pair-production $\chi_{1} \bar{\chi}_{1}$ (both on-shell) followed by the decay of one of the on-shell $\chi$ to $t W$, and, (ii) the single resonant (SR) channel including $\chi_{1} \bar{\chi}_{1}^{*}$ (one of the $\chi$ off-shell), and in addition, the strict single-production of $\chi_{1}$ shown in (b). We include both DR and SR and focus on 


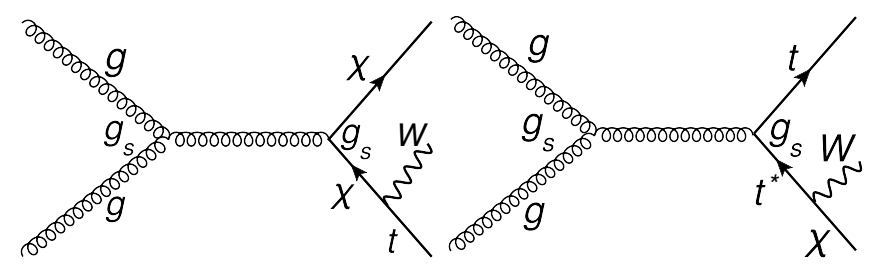

(a)

(b)

Figure 12. Sample Feynman diagrams contributing to the $p p \rightarrow \chi_{1} t W$ process. In (a) when both the $\chi$ 's are on-shell, we have a DR contribution, while when one of them is off-shell we have the $\mathrm{SR}$ process; the other contribution to SR comes from strict single production diagrams like the one shown in (b).

the channel

$$
p p \rightarrow \chi_{1} t W \rightarrow t W t W \rightarrow t W t \ell \nu .
$$

If the $W$ 's (including the ones coming from the tops) decay hadronically, then the signature for this channel would be $b b \ell E_{T}+$ jets. If the tops can be reconstructed, then the main $\mathrm{SM}$ background for this signature would be $p p \rightarrow t t+$ jets, $t t V+$ jets, $t t V V+$ jets (where $V=\{W, Z\}), t t h+$ jets etc. In addition to the tops, if the hadronically decaying $W$ is also reconstructed, then $p p \rightarrow t W t W$ becomes the dominant background. Therefore, for the background we consider the SM process $p p \rightarrow t W t W \rightarrow t W t \ell \nu$. We consider it at the $t W t W$ level keeping in mind that the top-jets can be tagged with high efficiency using advanced top-tagging algorithms. We have discussed this issue in $\mathrm{C}$ in more detail. We obtain the signal and background cross-sections at the $t t W \ell \nu$ level i.e., only one $W$ decays leptonically. We perform our analysis at this level because for the signal we expect the lepton coming from the $W$ to have large $p_{T}$, whereas it is less probable for the background to have a high $p_{T}$ lepton. This feature of the lepton can be used to isolate the signal from the background. The lepton can be used as a trigger. We consider the $b b 6 j \ell E_{T}$ final state where $j$ includes only "light" jets $(u, d, c, s)$ and $\ell$ includes $e$ and $\mu$. From the $t W t \ell \nu$ level cross-section, we compute the rate for the final-state of interest by multiplying with appropriate branching ratios.

In order to select the signal while suppressing the background, we apply the following "basic" and "discovery" cuts and present the signal and the background cross sections in table 6 (table 7 ) for the $14 \mathrm{TeV}(8 \mathrm{TeV}) \mathrm{LHC}$ :

\section{Basic}

(a) $|y(\ell)| \leq 2.5$

(b) $p_{T}(\ell) \geq 10 \mathrm{GeV}$

\section{Discovery}
(a) $|y(\ell)| \leq 2.5$
(b) $p_{T}(\ell) \geq 125 \mathrm{GeV}$
(c) $p_{T}(W) \geq 250 \mathrm{GeV}$. 
The second set of cuts is chosen to optimize the signal over background ratio. It is our "discovery cut" motivated by the fact that in the signal, there are two high- $p_{T} W$ 's present at the $t t W W$ level and one of them decays to a high- $p_{T}$ lepton. To account for the various efficiencies we multiply both signal and background cross sections with a factor

$$
\eta_{\chi_{1}}=\left(\epsilon_{\mathrm{tag}}^{b}\right)^{2} \times\left(\epsilon_{\mathrm{rec}}^{W}\right)^{3} \times\left(\epsilon_{\mathrm{rec}}^{t}\right)^{2} \times\left(B R_{W \rightarrow j j}\right)^{3} \approx 0.082
$$

where $\epsilon_{\text {tag }}^{b}$ is the $b$-tagging efficiency, $\epsilon_{\text {rec }}^{W}$ is the $W$ reconstruction efficiency from $j j, \epsilon_{\text {rec }}^{t}$ is the $t$ reconstruction efficiency from $b W$. Combinatorics might be an important issue for reconstruction but at our level of analysis we ignore this complication. We take $\epsilon_{\mathrm{tag}}^{b}=0.5$, $\epsilon_{\text {rec }}^{t}=1, \epsilon_{\text {rec }}^{W}=1$ and $W \rightarrow j j$ branching ratio $B R_{W \rightarrow j j}=0.69$. As explained earlier, we then compute $\mathcal{L}_{5}$ for $5 \sigma$ significance and $\mathcal{L}_{10}$ for obtaining 10 signal events, and the larger of $\mathcal{L}_{5}$ and $\mathcal{L}_{10}$ is the discovery luminosity. In $\mathrm{C}$ we present a more sophisticated analysis by including additional 2 -jets background and identify cuts that can bring them under control without sacrificing the signal much. The $\kappa$ can be probed by isolating the SR contribution. Typically, for the range of the coupling arising in warped models, the contribution of the second type of diagrams shown in figure 12(b) to the total cross-section is very small. This means the $t W$ pair in the SR production of $\chi_{1}$ is dominantly coming from an off-shell heavy quark - $\chi_{1}^{*}$. At the $\chi_{1} t W$ level we isolate the SR contribution by applying only the kinematical cut on the invariant mass $M(t W)$,

$$
\left|M(t W)-M_{\chi_{1}}\right| \geq \alpha_{\text {cut }} M_{\chi_{1}} ; \alpha_{\text {cut }}=0.05,
$$

which ensures that the $t$ quark and the $W$ do not reconstruct to an on-shell $\chi_{1}$, i.e. this cut removes the DR contribution. To understand why the cross-section after the $\alpha_{\text {cut }}$ scales as $\kappa^{2}$, let us consider the $\kappa$ dependent part of the cross-section (from the type of diagram in figure $12(\mathrm{a})$ ),

$$
\sigma_{\chi_{1} t W} \propto \frac{\kappa^{2}}{\left(p^{2}-M_{\chi_{1}}^{2}\right)^{2}+\Gamma_{\chi_{1}}^{2} M_{\chi_{1}}^{2}},
$$

where $p$ is the momentum carried by the internal $\chi_{1}$. The cut of eq. (5.3) is chosen such that $\left|p^{2}-M_{\chi_{1}}^{2}\right|$ dominates over $\Gamma_{\chi_{1}} M_{\chi_{1}}$, and one can neglect $\Gamma_{\chi_{1}} M_{\chi_{1}}$ compared to $\left|p^{2}-M_{\chi_{1}}^{2}\right|$, ensuring that $\sigma_{\chi_{1} t W}$ scales as $\kappa^{2}$. In figure 13 we show the $t W$ invariant mass distribution for the $p p \rightarrow \chi_{1} t W$ process, and in table 5 the cross section before and after the $\alpha_{\text {cut }}$. We observe that the total cross section before the cut is almost constant but decrease slightly with increasing $\kappa$ due to finite width effects. The contribution in the off-shell region increases with $\kappa$ since the total width grows as $\kappa^{2}$ which makes the Breit-Wigner distributions wider. ${ }^{4}$ This is seen more quantitatively in table 5 , where the cross section after the $\alpha_{\text {cut }}$ scales as $\kappa^{2}$ (for $\kappa$ not too large). As $\kappa$ increases, the $\sigma_{\chi_{1} t W}$ value after the $\alpha_{\text {cut }}$ cannot increase arbitrarily as it remains bounded by the $\sigma_{\chi_{1} t W}$ value before the cut. This can be seen by keeping in mind that $\Gamma_{\chi_{1}}$ depends on $\kappa$, and from the fact that for a fixed value of $\alpha_{\text {cut }}$, the $\kappa^{2}$ scaling behavior of $\sigma$ breaks down as $\Gamma_{\chi_{1}}$ increases with increasing $\kappa$ and at some point the $\Gamma_{\chi_{1}} M_{\chi_{1}}$ term in the denominator starts dominating again. Therefore, to be sensitive to $\sigma_{S R}$, the choice of $\alpha_{\text {cut }}$ is crucial (see ref. [81] for more

\footnotetext{
${ }^{4} \mathrm{~A}$ similar plot for a $b^{\prime}$ is shown in ref. [81].
} 


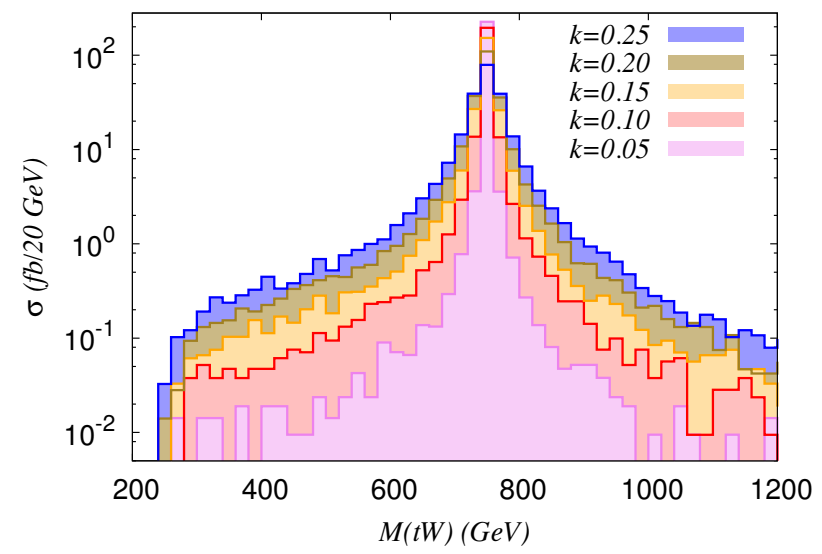

Figure 13. The $t W$ invariant mass distributions for the $p p \rightarrow \chi_{1} t W$ process for different $\kappa_{\chi_{1 R} t_{1 R} W}$ (denoted as $\kappa$ ), for $M_{\chi_{1}}=750 \mathrm{GeV}$ at the $14 \mathrm{TeV}$ LHC.

\begin{tabular}{|c|c|c|}
\hline$\kappa_{\chi_{1 R} t_{1 R} W}$ & $\begin{array}{c}\sigma\left(p p \rightarrow \chi_{1} t W\right) \\
(\mathrm{fb}) \text { before cut }\end{array}$ & $\begin{array}{c}\sigma\left(p p \rightarrow \chi_{1} t W\right) \\
(\mathrm{fb}) \text { after cut }\end{array}$ \\
\hline 0.05 & 239.37 & 4.945 \\
\hline 0.10 & 238.91 & 21.09 \\
\hline 0.15 & 236.31 & 45.92 \\
\hline 0.20 & 233.52 & 79.71 \\
\hline 0.25 & 229.40 & 118.71 \\
\hline
\end{tabular}

Table 5. Scaling behavior of $p p \rightarrow \chi_{1} t W$ single production cross-sections after the invariant mass cut defined in eq. (5.3), for $M_{\chi_{1}}=750 \mathrm{GeV}$ at the $14 \mathrm{TeV}$ LHC.

details). Taking $\alpha_{\text {cut }}$ too small will spoil the scaling because of the contamination from the pair production, but it should not be too large either as that will make the cross-section very small. In the warped $Z b \bar{b}$ protected model (ST and TT models), the $\kappa$ of eq. (3.1) are given in eqs. (2.23) and (2.33) and shown in figure 2 and table 1 respectively. For all $M_{\chi}$ considered here, we find $\mathcal{L}_{5}<\mathcal{L}_{10}$, and therefore in table 6 we present only $\mathcal{L}_{10}$. From table 6 we find that using $\sigma_{\text {tot }}$, i.e. including both SR and DR, the $14 \mathrm{TeV}$ LHC can probe $M_{\chi_{1}}$ up to $1.5 \mathrm{TeV}(1.75 \mathrm{TeV})$ with $100 \mathrm{fb}^{-1}\left(300 \mathrm{fb}^{-1}\right)$ of integrated luminosity for the ST model. The numbers in table 6 show that for the parameter ranges we are interested in, the $p p \rightarrow \chi_{1} t W$ process is dominated by the DR production. Hence, we do not display the cross sections and discovery luminosity separately for the TT model as the difference between them is only due the SR production (which depends on the $\kappa_{\chi_{1} t W}$ coupling).

As mentioned, the $\kappa$ can be probed by isolating the SR contribution. To present our results model-independently such that it is useful for other models with a $\chi t W$ coupling, we show in figure 14 the luminosity requirement $\left(\mathcal{L}_{D}\right)$ to observe the $p p \rightarrow \chi_{1} t W$ SR production process assuming the $\chi_{1} \rightarrow t W \mathrm{BR}$ to be $100 \%$, where, $\mathcal{L}_{D}=\operatorname{Max}\left(\mathcal{L}_{5}, \mathcal{L}_{10}\right)$. The blue and green dots show the reach for the SR process for the warped ST and TT models respectively. Although we compute $\mathcal{L}_{D}$ at the $\chi t W$ level multiplied by the appropriate 


\begin{tabular}{|c|c|c|c|c|c|c|c|}
\hline $\mathcal{X}$ & $\begin{array}{c}M_{\chi} \\
(\mathrm{GeV})\end{array}$ & $\begin{array}{l}\sigma_{\text {tot }} \\
(f b)\end{array}$ & $\begin{array}{c}\sigma_{S R} \\
(f b)\end{array}$ & cuts & $\begin{array}{c}\mathrm{S} \\
(f b)\end{array}$ & $\begin{array}{l}\text { BG } \\
(f b)\end{array}$ & $\begin{array}{c}\mathcal{L} \\
\left(f b^{-1}\right)\end{array}$ \\
\hline \multirow[t]{2}{*}{$\mathcal{X}_{1}$} & \multirow[t]{2}{*}{500} & \multirow[t]{2}{*}{2566} & \multirow[t]{2}{*}{261.5} & Basic & 977.5 & 3.257 & - \\
\hline & & & & Disc. & 146.1 & 0.115 & 0.826 \\
\hline \multirow[t]{2}{*}{$\mathcal{X}_{2}$} & \multirow[t]{2}{*}{750} & \multirow[t]{2}{*}{260.0} & \multirow[t]{2}{*}{29.31} & Basic & 99.99 & 3.257 & - \\
\hline & & & & Disc. & 42.74 & 0.115 & 2.824 \\
\hline \multirow[t]{2}{*}{$\mathcal{X}_{3}$} & \multirow[t]{2}{*}{1000} & \multirow[t]{2}{*}{46.47} & \multirow[t]{2}{*}{5.198} & Basic & 17.92 & 3.257 & - \\
\hline & & & & Disc. & 11.36 & 0.115 & 10.63 \\
\hline \multirow[t]{2}{*}{$\mathcal{X}_{4}$} & \multirow[t]{2}{*}{1250} & \multirow[t]{2}{*}{11.22} & \multirow[t]{2}{*}{1.231} & Basic & 4.305 & 3.257 & - \\
\hline & & & & Disc. & 3.226 & 0.115 & 37.42 \\
\hline \multirow[t]{2}{*}{$\mathcal{X}_{5}$} & \multirow[t]{2}{*}{1500} & \multirow[t]{2}{*}{3.242} & \multirow[t]{2}{*}{0.364} & Basic & 1.235 & 3.257 & - \\
\hline & & & & Disc. & 1.010 & 0.115 & 119.5 \\
\hline \multirow[t]{2}{*}{$\mathcal{X}_{6}$} & \multirow[t]{2}{*}{1750} & \multirow[t]{2}{*}{1.040} & \multirow[t]{2}{*}{0.121} & Basic & 0.393 & 3.257 & - \\
\hline & & & & Disc. & 0.339 & 0.115 & 355.8 \\
\hline
\end{tabular}

Table 6. Signal (S) and background (BG) cross sections (in $f b$ ) for $p p \rightarrow \chi t W \rightarrow t t W \ell \nu$ channel at the $14 \mathrm{TeV}$ LHC for the ST model. For the BG we have considered $p p \rightarrow t t W \ell \nu$ process within the SM. The $\mathcal{X}_{i}$ 's correspond to the parameter sets detailed in table 1 . The luminosity requirement $(\mathcal{L})$ is computed using $\sigma_{\text {tot }}$ after including the factor $\eta_{\chi_{1}}$ defined in eq. (5.2). The $\sigma_{\text {tot }}$ is computed at the $\chi_{1} t W$ level with no cut applied. $\sigma_{S R}$ is computed at the $\chi t W$ level with only an invariant mass cut applied on $t W$ as defined in eq. (5.3).

\begin{tabular}{|c|c|c|c|c|c|c|c|}
\hline $\mathcal{X}$ & $\begin{array}{c}M_{\chi} \\
(\mathrm{GeV})\end{array}$ & $\begin{array}{c}\sigma_{\text {tot }} \\
(f b)\end{array}$ & $\begin{array}{c}\sigma_{S R} \\
(f b)\end{array}$ & cuts & $\begin{array}{c}\mathrm{S} \\
(f b)\end{array}$ & $\begin{array}{c}\mathrm{BG} \\
(f b)\end{array}$ & $\begin{array}{c}\mathcal{L} \\
\left(f b^{-1}\right)\end{array}$ \\
\hline $\mathcal{X}_{1}$ & 500 & 374.2 & 36.63 & Basic & 144.0 & 0.622 & - \\
\cline { 5 - 8 } & & & & Disc. & 18.40 & 0.011 & 6.560 \\
\hline $\mathcal{X}_{2}$ & 750 & 25.61 & 2.741 & Basic & 9.927 & 0.622 & - \\
\cline { 5 - 8 } & & & & Disc. & 4.103 & 0.011 & 29.42 \\
\hline $\mathcal{X}_{3}$ & 1000 & 2.817 & \multirow{2}{*}{0.315} & Basic & 1.092 & 0.622 & - \\
\cline { 5 - 8 } & & & Disc. & 0.680 & 0.011 & 177.5 \\
\hline $\mathcal{X}_{4}$ & 1250 & \multirow{2}{*}{0.381} & \multirow{2}{*}{0.042} & Basic & 0.147 & 0.622 & - \\
\cline { 5 - 8 } & & & & Disc. & 0.109 & 0.011 & 1105 \\
\hline
\end{tabular}

Table 7. Same as in table 6 for the $8 \mathrm{TeV}$ LHC.

BRs, with only the invariant mass cut of eq. (5.3), we expect that the inclusion of the full decays and the basic and discovery cuts should change $\mathcal{L}_{D}$ only by a small amount. Here we vary $\kappa_{\chi_{1 R} t_{1 R} W}$ keeping the other coupling $\kappa_{\chi_{1 L} t_{1 L} W}$ zero (since this is the case in the ST and TT models). The plot will look identical if we instead vary $\kappa_{\chi_{1 L} t_{1 L} W}$ keeping $\kappa_{\chi_{1 R} t_{1 R} W}=0$. The background for the $\chi_{1} t W$ SR production is computed at the $t W t W$ level after demanding that any one of the $t W$ pair satisfies the cut defined in eq. (5.3). 


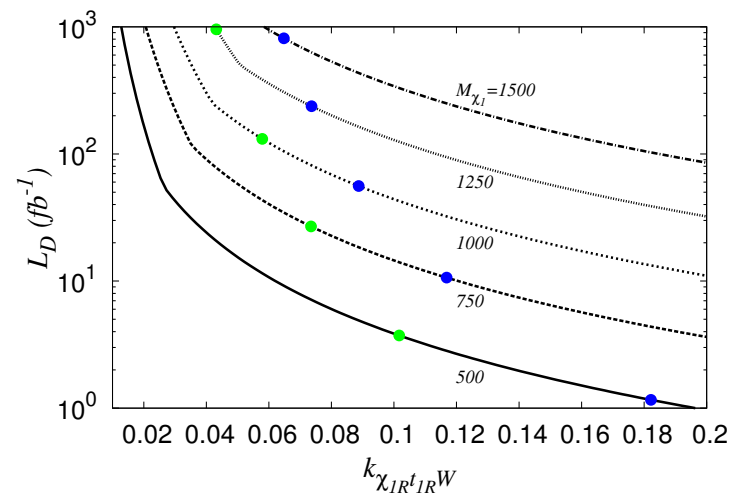

Figure 14. Luminosity requirements $\left(\mathcal{L}_{D}\right.$, in $\left.f b^{-1}\right)$ for observing the $p p \rightarrow \chi_{1} t W$ single resonant (SR) channel as functions of $\kappa_{\chi_{1 R} t_{1 R} W}$ for different $M_{\chi_{1}}$ (in GeV) at the $14 \mathrm{TeV}$ LHC. $\mathcal{L}_{D}$ is computed after including all BRs and $b$-tagging efficiency. The blue and green dots correspond to the ST and TT models respectively.

This can be expressed as

$$
\left|M\left(t_{1} W_{i}\right)-M_{\chi_{1}}\right| \geq \alpha_{\mathrm{cut}} M_{\chi_{1}} \quad \mathrm{AND} \quad\left|M\left(t_{2} W_{j}\right)-M_{\chi_{1}}\right| \leq \alpha_{\mathrm{cut}} M_{\chi_{1}}
$$

where $t$ 's and $W$ 's are $p_{T}$-ordered and $i, j=\{1,2\}$ with $i \neq j$. The kinks in the graphs appear because of the transition from $\mathcal{L}_{5}$ to $\mathcal{L}_{10}$ along the increasing values of the coupling. For getting the SR reach in the warped model, tables 6 and 7 give the SR cross-section $\sigma_{S R}$ for the ST model.

Finally, we note that there are other single production channels for $\chi_{1}$ at the LHC like the $W^{ \pm}$mediated $p p \rightarrow \chi_{1} t$ or $p p \rightarrow \chi_{1} t q$ (studied in ref. [34] in the context of composite Higgs models). However, unlike the $p p \rightarrow \chi_{1} t W$ process, these are electroweak processes due to which we find their cross-sections to be much smaller. Also, we expect $\sigma\left(\chi_{2} \chi_{2}\right)<\sigma\left(\chi_{1} \chi_{1}\right)$ due to the larger $M_{\chi_{2}}$, and since already the $\chi_{1}$ pair-production is signal rate limited, we do not explore the $\chi_{2}$ production and the subsequent $\chi_{2} \rightarrow \chi_{1} h$ or $\chi_{2} \rightarrow \chi_{1} Z$ channels.

\section{$5.2 \quad t^{\prime}$ LHC signatures}

At the LHC, apart from the usual pair production channel, a charge $2 / 3$ vectorlike $t_{2}$ can be produced through the following single production channels

$$
p p \rightarrow t_{2} W, t_{2} b, t_{2} t, t_{2} b W, t_{2} t Z, t_{2} t h .
$$

In models where the $t_{2} b W$ coupling is much smaller than the others (as for instance in the warped ST and TT models), we can ignore the single production channels $t_{2} W, t_{2} b, t_{2} b W$ channels. We parametrize the $t_{2} t Z$ and $t_{2} t h$ interaction terms model-independently as shown in eq. (3.2).

Similar to the discussion for the $\chi$ in section 5.1, here too we identify the double resonant (DR) and single resonant (SR) channels, and consider the thth final state. As 


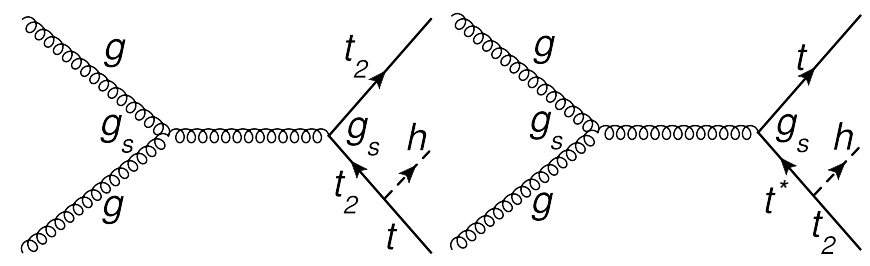

(a)

(b)

Figure 15. Sample Feynman diagrams contributing to the $p p \rightarrow t_{2}$ th process. In (a) when both the $\chi$ 's are on-shell, we have a DR contribution, while when one of them is off-shell we have the SR process; the other contribution to SR comes from the strict single production diagrams like the one shown in (b).

shown in figure 15, this includes (i) the double resonant (DR) pair-production $t_{2} \bar{t}_{2}$ (both on-shell) followed by the decay of one of the on-shell $t_{2} \rightarrow t h$, (ii) the single resonant (SR) channel including $t_{2} \bar{t}_{2}^{*}$ (one of the $t_{2}$ off-shell), and in addition, the single-production of $t_{2}$. We therefore include DR and SR and consider the process

$$
p p \rightarrow t_{2} t h \rightarrow t h t h \rightarrow t b b t b b,
$$

and focus on the $6 b+4 j$ final-state, where $j$ includes only "light" jets $(u, d, c, s)$. We obtain the signal cross-sections at the tbbtbb level and multiply by appropriate branching ratios relevant to the above final state. We take the Higgs boson mass to be $125 \mathrm{GeV}$ in all our computations. We assume a $b$-tagging efficiency $\epsilon_{\text {tag }}^{b}=0.5$, and demand only four of the six $b$-jets to be $b$-tagged (ref. [82] also follows a similar approach) to get a better signal rate. We require the two top-quarks to be reconstructed from two $b$-tagged jets and four $J$ (where $J$ stands for either a light-jet or an untagged $b$-jet) and then the two $h$ to be reconstructed from the remaining two $b$-tagged jets and two $J$. Here we do not deal with any complications of combinatorics. For this channel the main SM background come from $p p \rightarrow t t V_{h}+$ jets, $t t V_{h} V_{h}+$ jets (where $V_{h}=\{W, Z, h\}$ ) processes. We compute the background cross-sections at the ttbbJJ level, i.e. $p p \rightarrow t t b b J J$ process which includes all tree level SM processes leading to $t$ tbbJ $J$ final state. However, due to requiring the four jets to reconstruct to the two $h$ by applying the invariant mass cuts, the SM QCD contribution to the $p p \rightarrow t t b b J J$ process becomes negligible and the dominant $\mathrm{SM}$ background contribution comes from the $p p \rightarrow t t h h$ process. To keep most of the signal events while suppressing the background, we apply the following "basic" and "discovery" cuts on the ttbbJJ events:

\section{Basic}
(a) $|y(J)| \leq 2.5$
(b) $\Delta R(J J) \geq 0.4$
(c) $p_{T}(J) \geq 25 \mathrm{GeV}$

\section{Discovery}

(a) $|y(J)| \leq 2.5$ 
(b) $\Delta R(J J) \geq 0.4$

(c) For $p_{T}$ ordered jets:

$$
p_{T}^{1 \text { st }}(J), p_{T}^{2 \text { nd }}(J) \geq 175 \mathrm{GeV} \text { and } p_{T}^{3 \text { rd }}(J), p_{T}^{4 \text { th }}(J) \geq 25 \mathrm{GeV}
$$

(d) $\left|M\left(J_{i}, J_{j}\right)-m_{h}\right| \leq 10 \mathrm{GeV}$ and $\left|M\left(J_{k}, J_{l}\right)-m_{h}\right| \leq 10 \mathrm{GeV}$ where $i \neq j \neq k \neq l$.

where $\Delta R(i j)=\sqrt{\Delta \phi_{i j}^{2}+\Delta \eta_{i j}^{2}}$ is the angular separation between any two jets, $\phi$ is the azimuthal angle and $\eta$ is the pseudo-rapidity. The "discovery cut" is motivated by the fact that for the signal, there is at least one high- $p_{T}$ Higgs coming from the heavy $t_{2}$ decay, and we expect the $b$-quarks coming from the Higgs decay to have a large $p_{T}$. We multiply both signal and background cross sections with a factor

$$
\eta_{t_{2}}=\left(\epsilon_{\mathrm{tag}}^{b}\right)^{4} \times\left(\epsilon_{\mathrm{rec}}^{W}\right)^{2} \times\left(\epsilon_{\mathrm{rec}}^{t}\right)^{2} \times\left(B R_{W \rightarrow j j}\right)^{2} \approx 0.0299
$$

where we take $\epsilon_{\text {tag }}^{b}=0.5, \epsilon_{\text {rec }}^{t}=1, \epsilon_{\text {rec }}^{W}=1$ and $W \rightarrow j j$ branching ratio $B R_{W \rightarrow j j}=0.69$.

In the warped models detailed in section 2 , the $t_{2} b W$ coupling (i.e. $\kappa_{t_{2} b W}$ ) becomes very small for heavy $t_{2}$ as explained in section 3 . As a result, the production cross sections for the $p p \rightarrow t_{2} W, t_{2} b, t_{2} b W$ channels are small compared to the rest of the single production channels. Among the other channels, the $p p \rightarrow t_{2} t$ channel is weak interaction mediated ${ }^{5}$ (the $t_{2} t$ pair actually comes from an off-shell $Z$ or $h$ ) and so is less significant than the $p p \rightarrow t_{2} t Z$ or $p p \rightarrow t_{2} t h$ channels, and we do not consider the former due to the small $B R_{Z \rightarrow \ell \ell}$. Thus in the warped models, the $p p \rightarrow t_{2} t h$ channel that we have focused on is a promising channel. As already mentioned, the $t_{2}$ in the warped model without $Z b \bar{b}$ protection (DT model) is very heavy making its discovery very challenging. We therefore do not consider further the $t^{\prime}$ in the DT model. The $\kappa$ in the warped models with $Z b \bar{b}$ protection (ST and TT models) are given in section 2. We present our results for the ST model at the $14 \mathrm{TeV}(8 \mathrm{TeV})$ LHC in table 8 (table 9) after the cuts shown above. Defining as before, $\mathcal{L}_{5 \sigma}$ as the Luminosity for $S / \sqrt{B}=5$ and $\mathcal{L}_{10}$ that for 10 events, we find that $\mathcal{L}_{5 \sigma}<\mathcal{L}_{10}$ in most of parameter-space, except for $M_{t_{2}}=1250 \mathrm{GeV}$ for $14 \mathrm{TeV}$ LHC, and we present the maximum of $\mathcal{L}_{5 \sigma}$ and $\mathcal{L}_{10}$ in table 8. From $\sigma_{\text {tot }}=\sigma_{D R}+\sigma_{S R}$, we find that the $14 \mathrm{TeV} \mathrm{LHC}$ can probe $M_{t_{2}}$ of the order of $1 \mathrm{TeV}$ with $100 \mathrm{fb}^{-1}$ of integrated luminosity in the ST model.

As mentioned earlier, the SR process can give important information on the electroweak couplings $\kappa$ (while the DR depends dominantly on $g_{S}$ ). To explore this aspect, we compute the $p p \rightarrow t_{2} t h$ SR production cross-sections from the $p p \rightarrow t_{2} t h$ signal events by applying the kinematical cut

$$
\left|M(t h)-M_{t_{2}}\right| \geq \alpha_{\text {cut }} M_{t_{2}} ; \alpha_{\text {cut }}=0.05 .
$$

The background for the $t_{2} t h \mathrm{SR}$ production is computed at the thth level after demanding that any one of the th pairs satisfies the invariant mass cut defined in eq. (5.9). This cut can be expressed as

$$
\left|M\left(t_{1} h_{i}\right)-M_{t_{2}}\right| \geq \alpha_{\text {cut }} M_{t_{2}} \quad \text { AND } \quad\left|M\left(t_{2} h_{j}\right)-M_{t_{2}}\right| \leq \alpha_{\text {cut }} M_{t_{2}}
$$

\footnotetext{
${ }^{5}$ However, this could also arize from the decay of the KK Gluon; see ref. [44].
} 


\begin{tabular}{|c|c|c|c|c|c|c|c|}
\hline $\mathcal{T}$ & $\begin{array}{c}M_{t_{2}} \\
(\mathrm{GeV})\end{array}$ & $\begin{array}{l}\sigma_{\text {tot }} \\
(f b)\end{array}$ & $\begin{array}{l}\sigma_{S R} \\
(f b)\end{array}$ & cuts & $\begin{array}{c}\mathrm{S} \\
(f b)\end{array}$ & $\begin{array}{l}\text { BG } \\
(f b)\end{array}$ & $\begin{array}{c}\mathcal{L} \\
\left(f b^{-1}\right)\end{array}$ \\
\hline \multirow[t]{2}{*}{$\mathcal{T}_{1}$} & \multirow[t]{2}{*}{500} & \multirow[t]{2}{*}{1247} & \multirow[t]{2}{*}{223.0} & Basic & 237.4 & 102.7 & - \\
\hline & & & & Disc. & 52.38 & 0.389 & 6.379 \\
\hline \multirow[t]{2}{*}{$\mathcal{T}_{2}$} & \multirow[t]{2}{*}{750} & \multirow[t]{2}{*}{122.3} & \multirow[t]{2}{*}{18.30} & Basic & 22.67 & 102.7 & - \\
\hline & & & & Disc. & 13.25 & 0.389 & 25.22 \\
\hline \multirow[t]{2}{*}{$\mathcal{T}_{3}$} & \multirow[t]{2}{*}{1000} & \multirow[t]{2}{*}{20.33} & \multirow[t]{2}{*}{2.715} & Basic & 3.088 & 102.7 & - \\
\hline & & & & Disc. & 2.421 & 0.389 & 138.0 \\
\hline \multirow[t]{2}{*}{$\mathcal{T}_{4}$} & \multirow[t]{2}{*}{1250} & \multirow[t]{2}{*}{4.444} & \multirow[t]{2}{*}{0.590} & Basic & 0.477 & 102.7 & - \\
\hline & & & & Disc. & 0.415 & 0.389 & 1889.2 \\
\hline
\end{tabular}

Table 8. Signal (S) and background (BG) cross sections (in $f b$ ) for $p p \rightarrow t_{2} t h \rightarrow t t b b b b$ channel at the $14 \mathrm{TeV}$ LHC for the ST model. For the BG we have considered the SM $p p \rightarrow t t b b J J$ process where the dominant contribution comes from $p p \rightarrow t t h h$. The $\mathcal{T}_{i}$ 's correspond to the parameter sets detailed in table 2. The luminosity requirement $\mathcal{L}$ is computed using $\sigma_{\text {tot }}$ after including the factor $\eta_{t_{2}}$ defined in eq. (5.8). These numbers are obtained using $B R_{h \rightarrow b b}=0.8$. The $\sigma_{\text {tot }}=\sigma_{D R}+\sigma_{S R}$ is computed at the $t_{2} t h$ level with no cut applied, whereas $\sigma_{S R}$ is computed at the $t_{2} t h$ level with only the $t W$ invariant mass cut of eq. (5.9) applied.

\begin{tabular}{|c|c|c|c|c|c|c|c|}
\hline $\mathcal{T}$ & $\begin{array}{c}M_{t_{2}} \\
(\mathrm{GeV})\end{array}$ & $\begin{array}{c}\sigma_{\text {tot }} \\
(f b)\end{array}$ & $\begin{array}{c}\sigma_{S R} \\
(f b)\end{array}$ & cuts & $\begin{array}{c}\mathrm{S} \\
(f b)\end{array}$ & $\begin{array}{c}\mathrm{BG} \\
(f b)\end{array}$ & $\begin{array}{c}\mathcal{L} \\
\left(f b^{-1}\right)\end{array}$ \\
\hline $\mathcal{T}_{1}$ & 500 & 181.3 & 32.48 & Basic & 35.83 & 16.43 & - \\
\cline { 5 - 8 } & & & & Disc. & 6.702 & 0.035 & 49.85 \\
\hline $\mathcal{T}_{2}$ & \multirow{2}{*}{750} & 11.96 & \multirow{2}{*}{1.690} & Basic & 2.353 & 16.43 & - \\
\cline { 5 - 8 } & & & & Disc. & 1.325 & 0.035 & 252.3 \\
\hline $\mathcal{T}_{3}$ & 1000 & \multirow{2}{*}{1.222} & \multirow{2}{*}{0.168} & Basic & 0.206 & 16.43 & - \\
\cline { 5 - 8 } & & & Disc. & 0.162 & 0.035 & 2056.8 \\
\hline
\end{tabular}

Table 9. Same as in table 8 for the $8 \mathrm{TeV}$ LHC.

where $t$ 's and $h$ 's are $p_{T}$-ordered and $i, j=\{1,2\}$ with $i \neq j$. Just as in the case of $\chi_{1}$ production, for the parameter ranges we are interested in, $p p \rightarrow t_{2}$ th process is dominated by the DR production. We have also verified that with our choice of $\alpha_{\text {cut }}$ the $\sigma_{S R}$ scales as $\kappa_{t_{2} t h}^{2}$. Since the SR production can give us information about the off-diagonal $t_{2} t h$ coupling, in figure 16 we present model-independently the luminosity required for $p p \rightarrow t_{2}$ th $\mathrm{SR}$ production channel assuming $B R_{t_{2} \rightarrow t h}$ to be $100 \%$. In doing this we vary $\kappa_{t_{2 L} t_{1 R} h}$ keeping the other coupling $\kappa_{t_{1 L} t_{2 R} h}$ to zero (as is the case for instance in the warped model). We find that $p p \rightarrow t_{2}$ th events are signal rate limited (i.e., $\mathcal{L}_{10}>\mathcal{L}_{5}$ ) in the parameter range we have considered. In figure 16 we show the luminosity required for the warped ST model as blue dots and the TT model as green dots.

In the ST or TT models, for heavy $t_{2}$, the branching ratios for $t_{2} \rightarrow t h$ and $t_{2} \rightarrow t Z$ are comparable, i.e.,

$$
B R_{t_{2} \rightarrow t h} \approx B R_{t_{2} \rightarrow t Z}
$$




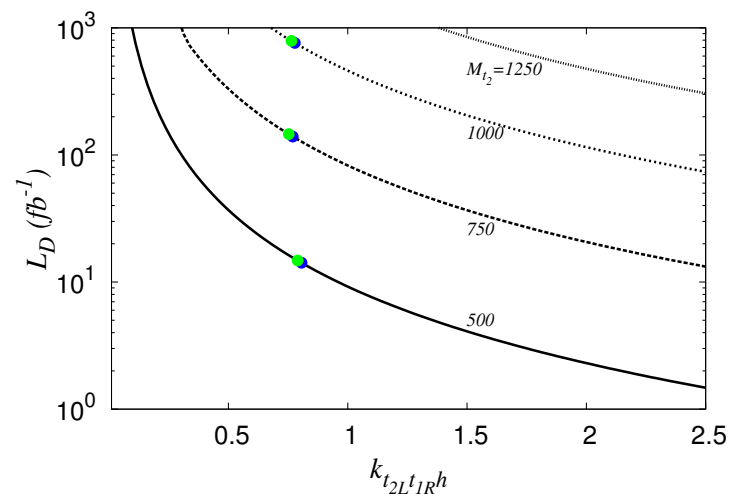

Figure 16. Luminosity requirements $\left(\mathcal{L}_{D}\right.$, in $\left.f b^{-1}\right)$ for observing the $p p \rightarrow t_{2} t h$ SR process as functions of $\kappa_{t_{2 L} t_{1 R} h}$ for different $M_{t_{2}}$ (in GeV) at the $14 \mathrm{TeV}$ LHC. The luminosity is computed after including all BRs and $b$-tagging efficiency. The blue and green dots correspond to the ST and TT models respectively.

Hence one could as well study the following processes:

$$
\begin{aligned}
& p p \rightarrow t_{2} \bar{t} h \rightarrow(t Z) t h \rightarrow b W Z b W h, \\
& p p \rightarrow t_{2} \bar{t} Z \rightarrow(t h) t Z \rightarrow b W h b W Z, \\
& p p \rightarrow t_{2} \bar{t} Z \rightarrow(t Z) t Z \rightarrow b W Z b W Z .
\end{aligned}
$$

Of these the first two can even lead to $4 b+6 j$ final states which is exactly what we have used for our analysis by demanding only $4 b$-tagged jets. We don't expect the LHC reach to be very different for these two channels from what we have estimated. This is because, the main difference between these two channels and what we have considered comes from the facts that the Higgs boson is a bit heavier than the $Z$ and $B R_{h \rightarrow b b}>B R_{Z \rightarrow J J}$. However for the last process, i.e. $p p \rightarrow t_{2} \bar{t} Z \rightarrow(t Z) t Z$, we cannot demand $4 b$-tagged jets anymore and as a result we must consider one of the $Z$ decaying leptonically to act as the trigger. Since $B R_{Z \rightarrow \ell \ell}<B R_{Z \rightarrow J J}$, in this case the signal rate will be quite small.

\section{$5.3 \quad b^{\prime}$ LHC signatures}

The important single production channels of a vectorlike $b^{\prime}$ were explored in ref. [50], which included $t b^{\prime}, b b^{\prime}, b^{\prime} h, b^{\prime} Z, q t b^{\prime}, q b b^{\prime}, b b^{\prime} Z, b b^{\prime} h, q b^{\prime} Z, q b^{\prime} h, t b^{\prime} W$ and $q b^{\prime} W$ processes. As mentioned earlier, ref. [45] studies the $b b^{\prime}$ production via KK-gluon. The $q t b^{\prime}$ process has been studied in ref. [34] in the context of composite Higgs models. A detailed study of the collider signatures and discovery reach for $b^{\prime}$ pair production and $b^{\prime} Z$ and $b^{\prime} h$ single production channels is already presented in a model independent manner in ref. [1]. Here we consider another $b^{\prime}$ single production process, thus adding to the study of ref. [1]. The process we consider is shown in figure 17 , namely

$$
p p \rightarrow b_{2} b Z \rightarrow b Z b Z,
$$

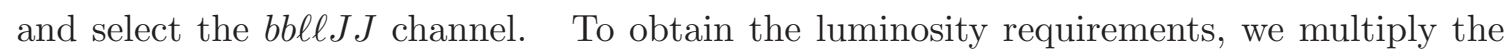




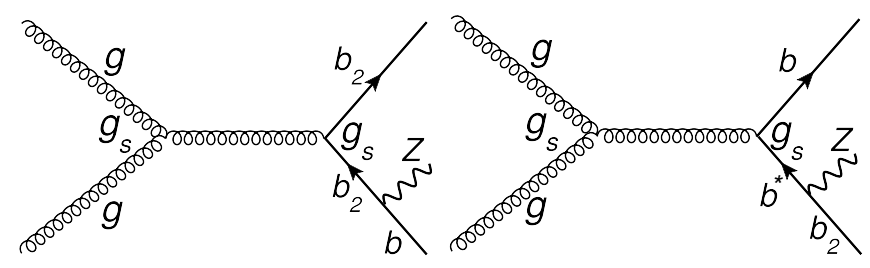

(a)

(b)

Figure 17. In (a) when both the $b_{2}$ are on-shell, we have a double resonant (DR) contribution, while when one of them is off-shell we have the single resonant (SR) process; the other contribution to SR coming from the strict single production diagram is shown in (b).

cross-section obtained at the $b Z b Z$ level by the factor

$$
\eta_{b_{2}}=2 \times\left(\epsilon_{\mathrm{tag}}^{b}\right)^{2} \times \epsilon_{\mathrm{rec}}^{(\ell \ell \rightarrow Z)} \times \epsilon_{\mathrm{rec}}^{(J J \rightarrow Z)} \times\left(B R_{Z \rightarrow J J}\right) \times\left(B R_{Z \rightarrow \ell \ell}\right) \approx 0.023,
$$

to take into account the various BR and efficiencies. Here $\epsilon_{\mathrm{rec}}^{(\ell \ell Z)}$ and $\epsilon_{\mathrm{rec}}^{(J J \rightarrow Z)}$ stand for reconstruction efficiency of $Z$ from $\ell \ell$ and $J J$ respectively. We take $\epsilon_{\operatorname{tag}}^{b}=0.5, \epsilon_{\text {rec }}^{(\ell \ell \rightarrow Z)}=1$ and $\epsilon_{\text {rec }}^{(J J \rightarrow Z)}=1$ and the branching ratios $B R_{Z \rightarrow J J}=0.69$ and $B R_{Z \rightarrow \ell \ell}=0.068$. The extra 2 factor appears because either of the $Z$ can decay to the $\ell \ell$ pair. We parametrize the $b_{2} b Z$ interaction terms model-independently as shown in eq. (3.3). Analogous to the previous subsections, we have both double resonant (DR) and single resonant (SR) contributions to the $b_{2} b Z$ final state. Isolating the $\mathrm{SR}$ contribution can give us information about the off-diagonal $b_{2} b Z$ couplings. To this end, we compute the $p p \rightarrow b_{2} b Z$ SR production crosssection from the $p p \rightarrow b_{2} b Z$ cross-section by applying the kinematical cut

$$
\left|M(b Z)-M_{b_{2}}\right| \geq \alpha_{\text {cut }} M_{b_{2}} ; \quad \alpha_{\text {cut }}=0.05 .
$$

We have also verified that with our choice of $\alpha_{\text {cut }}$ the $\sigma_{S R}$ scales as $\kappa_{b_{2} b Z}^{2}$. The main $\mathrm{SM}$ backgrounds for the $b_{2} b Z$ SR production come from $p p \rightarrow b b Z+$ jets, $b b Z V$ (where $V=\{W, Z\})$ processes. Applying invariant mass-cut around $Z$-mass one can significantly reduce $b b Z+$ jets and $b b Z W$ contributions. Therefore, we compute the background for the $b_{2} b Z$ SR production at the $b Z b Z$ level. We demand that any one of the $b Z$ pairs satisfies the invariant mass cut of eq. (5.17) i.e.

$$
\left|M\left(b_{1} Z_{i}\right)-M_{b_{2}}\right| \geq \alpha_{\text {cut }} M_{b_{2}} \quad \text { AND } \quad\left|M\left(b_{2} Z_{j}\right)-M_{b_{2}}\right| \leq \alpha_{\text {cut }} M_{b_{2}}
$$

where $b$ 's and $Z$ 's are $p_{T}$-ordered and $i, j=\{1,2\}$ with $i \neq j$. In figure 18 we present the luminosity requirement for $p p \rightarrow b_{2} b Z$ SR production channel in a model-independent manner assuming $B R_{b_{2} \rightarrow b Z}$ to be $100 \%$. The kinks in the graphs appear because of the transition from $\mathcal{L}_{5}$ to $\mathcal{L}_{10}$ along the increasing values of the coupling. In doing this we vary $\kappa_{b_{2 L} b_{1 L} Z}$ keeping the other coupling $\kappa_{b_{2 R} b_{1 R} Z}$ zero. (This is the case in the warped models we have considered.) In table 10 we compare the various SR channel cross-sections model-independently. The $b_{2} b Z$ cross-section is after applying the invariant mass cut of eq. (5.17), while the others are without any cuts. We see that the $b_{2} Z$ channel studied in ref. [1] and the $b_{2} b Z$ SR process studied here are comparable in signal cross-section; however the latter case requires larger luminosity since the background is larger. 


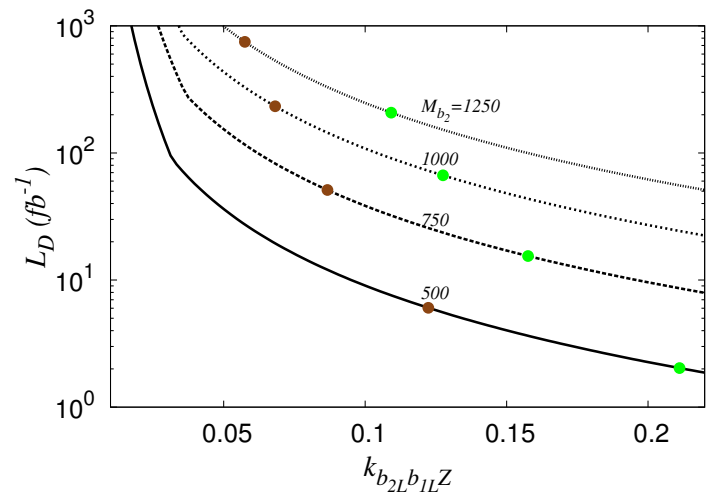

Figure 18. Luminosity requirements $\left(\mathcal{L}_{D}\right.$, in $\left.f b^{-1}\right)$ for observing the $p p \rightarrow b_{2} b Z$ SR process as functions of $\kappa_{b_{2 L} b_{1 L} Z}$ for different $M_{b_{2}}$ (in GeV) at the $14 \mathrm{TeV}$ LHC. $\mathcal{L}_{D}$ is computed after including all BRs and $b$-tagging efficiency as shown in eq. (5.16). The brown and green dots correspond to the DT and TT models respectively.

\begin{tabular}{|c|c|c|c|}
\hline$M_{b_{2}}(\mathrm{GeV})$ & $\sigma\left(p p \rightarrow b_{2} Z\right)(\mathrm{fb})$ & $\sigma\left(p p \rightarrow b_{2} b\right)(\mathrm{fb})$ & $\sigma\left(p p \rightarrow b_{2} b Z\right)(\mathrm{fb})$ \\
\hline 500 & 81.50 & 15.86 & 47.12 \\
\hline 750 & 16.67 & 3.910 & 11.10 \\
\hline 1000 & 4.630 & 1.256 & 3.933 \\
\hline 1250 & 1.534 & 0.472 & 1.722 \\
\hline 1500 & 0.565 & 0.193 & 0.804 \\
\hline
\end{tabular}

Table 10. SR production cross-sections of $b_{2}$ for different $M_{b_{2}}$ with $\kappa_{b_{2}} b Z=0.1$. The $b_{2} b Z$ crosssection is after applying the invariant mass cut of eq. (5.17), while the others are without any cuts.

In the warped models, a $b^{\prime}$ is present in the DT and TT models, and the $\kappa$ of eq. (3.3) are given in eqs. (2.6) and in section 2.2.2 respectively. The $\kappa$ for the DT and TT models are shown in figure 6 , and for the TT model in table 3 . We can infer the luminosity required for the DR process from ref. [1]. For the DT model in the $p p \rightarrow b^{\prime} \bar{b}^{\prime} \rightarrow b Z \bar{b} Z \rightarrow b \ell \ell b j j$ channel, the $14 \mathrm{TeV}$ LHC reach is about $1250 \mathrm{GeV}$ with about $500 \mathrm{fb}^{-1}$. For the TT model, the $B R\left(b^{\prime} \rightarrow b Z\right)$ is about a factor of two bigger compared to the DT model; hence the luminosity being signal-rate limited, is about half. Turning next to the SR process, the brown and green dots in figure 18 are for the DT and TT warped models respectively. The corresponding signal cross-sections are shown in table 11. In the TT model, for simplicity, we have focused only on the $b_{2}$ signatures, although the $b_{3}$ is quite close in mass; a more complete analysis can include the $b_{3}$ contributions also. Analogously, one can also look at the bhbh channel which we have not explored in this work. In the DT model, for the choice of benchmark parameters discussed in section 3, we have a reach of $M_{b_{2}}=1000 \mathrm{GeV}$ with about $250 \mathrm{fb}^{-1}$, and in the TT model it is about $M_{b_{2}}=1250 \mathrm{GeV}$ with about $250 \mathrm{fb}^{-1}$. 


\begin{tabular}{|c|c|c||c|c|c|}
\hline \multicolumn{3}{|c|}{ DT model } & \multicolumn{4}{|c|}{ TT model } \\
\cline { 2 - 6 }$M_{b_{2}}(\mathrm{GeV})$ & $\kappa_{b_{1 L} b_{2 L} Z}$ & $\sigma_{b_{2} b Z}(\mathrm{fb})$ & $\mathcal{B}$ & $M_{b_{2}}(\mathrm{GeV})$ & $\sigma_{b_{2} b Z}(\mathrm{fb})$ \\
\hline 500 & 0.122 & 70.49 & $\mathcal{B}_{1}$ & 500 & 210.05 \\
\hline 750 & 0.087 & 8.341 & $\mathcal{B}_{2}$ & 750 & 27.56 \\
\hline 1000 & 0.068 & 1.829 & $\mathcal{B}_{3}$ & 1000 & 6.394 \\
\hline 1250 & 0.057 & 0.569 & $\mathcal{B}_{4}$ & 1250 & 2.054 \\
\hline
\end{tabular}

Table 11. Cross-sections for the process $p p \rightarrow b_{2} b Z$ in the DT and TT models for different choices of $M_{b_{2}}$. The cross-sections are obtained after applying the invariant mass cut of eq. (5.17). The couplings for the TT model corresponding to the parameter sets labelled by $\mathcal{B}_{i}$ are shown in table 3 .

\section{Conclusions}

We present the phenomenology and LHC Signatures of colored vectorlike fermions $\chi$ (EM charge $5 / 3$ ), $t^{\prime}$ (EM charge 2/3) and $b^{\prime}$ (EM charge -1/3). Such fermions appear in many BSM extensions. We take warped extra-dimensional models as the motivating framework for our analysis. However, our analysis applies to other models that have such fermions, and we present our results model-independently wherever possible. Our focus is the phenomenology due to the mixing of SM fermions with the new vectorlike fermions induced by EWSB.

We identify the allowed decay modes of the vectorlike quarks, compute their partial widths and branching ratios. This guides us in identifying promising channels for discovery of these vectorlike quarks at the LHC. While pair production via the gluon coupling usually has the largest cross-section at the LHC for the range of parameters we consider, a particular focus is single production channels of these vectorlike quarks, which although challenging, can probe the EW structure of the BSM model.

We consider three different cases of warped models as motivating examples, differing in the fermion representations under $\mathrm{SU}(2)_{L} \otimes \mathrm{SU}(2)_{R} \otimes \mathrm{U}(1)_{X}$ gauge group. We label them by the representation $t_{R}$ appears in, namely, Doublet Top (DT), Singlet Top (ST) and Triplet Top (TT) models. The first, the DT model, does not have the $Z b \bar{b}$ coupling protected and has stronger constraints on it, while the ST and TT models have custodial protection of the $Z b \bar{b}$ coupling and have less severe constraints on them. More than one $\chi$, $t^{\prime}$ or $b^{\prime}$ can be present depending on the model, and they can mix among themselves and the SM quarks as a result of off-diagonal EWSB induced mass mixing terms. We identify the mass eigenstates by diagonalizing the mass matrix, and work out the couplings that are relevant to the LHC phenomenology we discuss.

At the LHC we have computed the signal cross-sections and the dominant SM background to $\chi, t^{\prime}$ and $b^{\prime}$ productions, and find the $8 \mathrm{TeV}$ and $14 \mathrm{TeV}$ LHC discovery reach. For the $\chi$, we identify $p p \rightarrow \chi t W \rightarrow t W t W$ in the $2 b 6 j \ell E_{T}$ channel as a promising one. The $p p \rightarrow \chi t W$ process has contributions from: (a) double resonant (DR) process $p p \rightarrow \chi \bar{\chi}$ followed by $\chi \rightarrow t W$ decay where both $\chi$ are on-shell, and, (b) the single resonant (SR) process $p p \rightarrow \chi t W$ where only one $\chi$ is on-shell. The DR process dominantly depends only on 
the strong coupling $g_{s}$, while the SR process is directly sensitive to the EW couplings and mixing effects, and its measurement would give valuable information on the EW structure of the underlying BSM theory. We show that by applying an invariant mass cut to remove one on-shell $\chi$, we can get sensitivity to the EW couplings. Including both SR and DR, we find that at the $14 \mathrm{TeV}$ LHC the reach is about $m_{\chi}=1750 \mathrm{GeV}$ with about $350 \mathrm{fb}^{-1}$. In the same vein, for the $t^{\prime}$, we study the process $p p \rightarrow t_{2} t h \rightarrow$ thth in the $2 j 6 b \ell E_{T}$ channel as a promising one, and find that the $14 \mathrm{TeV}$ LHC can probe of the order of $1 \mathrm{TeV}$ mass with about $150 \mathrm{fb}^{-1}$. For the $b^{\prime}$ we discuss the process $p p \rightarrow b_{2} b Z \rightarrow b Z b Z$ in the $2 j 2 b \ell^{+} \ell^{-}$channel, and infer that the $14 \mathrm{TeV}$ LHC reach is about $1250 \mathrm{GeV}$ with about $250 \mathrm{fb}^{-1}$ for the TT model.

\section{Acknowledgments}

We thank K. Agashe and A. Pomarol for valuable discussions.

\section{A Fermion profiles}

The fermion KK mode profiles read as [61]

$$
\begin{aligned}
& f^{(0)}(y)=\sqrt{\frac{(1-2 c) k \pi R}{e^{(1-2 c) k \pi R}-1}} e^{-c k y} \\
& f^{(n)}(y)=\frac{e^{k y / 2}}{N_{n}}\left[J_{\alpha}\left(\frac{m_{n}}{k} e^{k y}\right)+b_{\alpha}\left(m_{n}\right) Y_{\alpha}\left(\frac{m_{n}}{k} e^{k y}\right)\right] \quad(n=1,2, \ldots)
\end{aligned}
$$

where $\alpha=|c+1 / 2| . \quad J_{\alpha}$ and $Y_{\alpha}$ are the Bessel functions of order $\alpha$ of the first and the second kind respectively. These profiles satisfy the following orthonormality condition,

$$
\frac{1}{\pi R} \int_{0}^{\pi R} d y e^{k y} f^{(m)}(y) f^{(n)}(y)=\delta_{m n}
$$

from which one can determine the normalization, $N_{n} . b_{\alpha}\left(m_{n}\right)$ and $m_{n}$ are determined through the $\mathrm{BC}$ on the branes. For fermions obeying $(-,+) \mathrm{BC}$, which means

$$
\left.f^{(n)}(y)\right|_{y=0}=0 \text { and }\left.\left(\partial_{y}+c k\right) f^{(n)}(y)\right|_{y=\pi R}=0 .
$$

From these two equations one obtains the following condition

$$
b_{\alpha}\left(m_{n}\right)=-\frac{J_{\alpha}\left(\frac{m_{n}}{k}\right)}{Y_{\alpha}\left(\frac{m_{n}}{k}\right)}=-\frac{\left(c+\frac{1}{2}\right) J_{\alpha}\left(\frac{m_{n}}{k} e^{\pi k R}\right)+\left(\frac{m_{n}}{k} e^{\pi k R}\right) J_{\alpha}^{\prime}\left(\frac{m_{n}}{k} e^{\pi k R}\right)}{\left(c+\frac{1}{2}\right) Y_{\alpha}\left(\frac{m_{n}}{k} e^{\pi k R}\right)+\left(\frac{m_{n}}{k} e^{\pi k R}\right) Y_{\alpha}^{\prime}\left(\frac{m_{n}}{k} e^{\pi k R}\right)} .
$$

This condition can be solved numerically for $m_{n}$ and $b_{\alpha}\left(m_{n}\right)$. The first fermion KK mass $m_{1}$ for $(-,+) \mathrm{BC}$ as a function of $c$ is shown in figure 19 . 


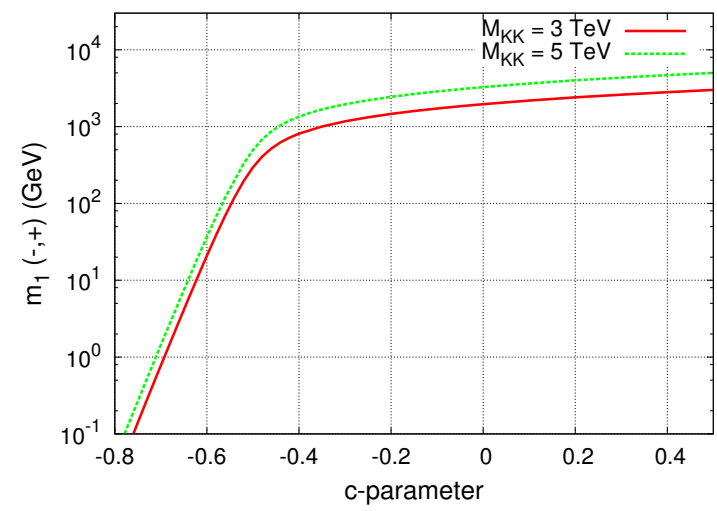

Figure 19. Masses of the first KK fermion with $(-,+) \mathrm{BC}$ as functions of $c$ for different values of the KK gauge boson masses.

\section{B $\quad t_{R}$ triplet case diagonalization}

Here we present analytical results for the mass matrix diagonalization and the resulting couplings in the mass basis in the limit of $m_{i j} / M_{\psi^{\prime}} \ll 1$ for the $t_{R}$ Triplet case (TT model) detailed in section 2.2.2.

The $m_{i j}$ in the charge $-1 / 3$ mass matrix in eq. (2.28) are the same, and defining $r_{b} \equiv m / M$, we find

$$
R_{L}^{T}=\frac{1}{\sqrt{1+2 r_{b}^{2}}}\left(\begin{array}{ccc}
-1 & r_{b} & r_{b} \\
0 & -\frac{\sqrt{1+2 r_{b}^{2}}}{\sqrt{2}} & \frac{\sqrt{1+2 r_{b}^{2}}}{\sqrt{2}} \\
\sqrt{2} r_{b} & \frac{1}{\sqrt{2}} & \frac{1}{\sqrt{2}}
\end{array}\right) ; \quad R_{R}=\left(\begin{array}{ccc}
1 & 0 & 0 \\
0 & -\frac{1}{\sqrt{2}} & \frac{1}{\sqrt{2}} \\
0 & \frac{1}{\sqrt{2}} & \frac{1}{\sqrt{2}}
\end{array}\right)
$$

with the mass eigenvalues $0, M, M \sqrt{1+2 r_{b}^{2}}$. The $b_{1}$ is identified as the SM b-quark, and the zero eigenvalue will be lifted when $\lambda_{b}$ terms are included.

The $Z$ boson neutral current interactions are (although not shown, the vector index on the gauge fields and the $\gamma^{\mu}$ between the fermion fields are implied)

$$
\begin{aligned}
\mathcal{L}_{N C}^{Z} \supset & g_{Z}\left\{\overline{b_{1 L}}\left[-\frac{1}{2}-s_{W}^{2} Q_{b}\right] b_{1 L}+\overline{b_{2 L}}\left[-\frac{1}{2}-s_{W}^{2} Q_{b}\right] b_{2 L}+\overline{b_{3 L}}\left[-\frac{1}{2}-s_{W}^{2} Q_{b}\right] b_{3 L}+\right. \\
& {\left[\overline{b_{1 L}}\left(\frac{\sqrt{2} r_{b}}{\sqrt{1+2 r_{b}^{2}}}\right)\left(-\frac{1}{2}\right) b_{2 L}+\overline{b_{2 L}}\left(\frac{1}{\sqrt{1+2 r_{b}^{2}}}\right)\left(-\frac{1}{2}\right) b_{3 L}+\text { h.c. }\right] } \\
& \overline{b_{1 R}}\left(-s_{W}^{2} Q_{b}\right) b_{1 R}+\overline{b_{2 R}}\left(-\frac{1}{2}-s_{W}^{2} Q_{b}\right) b_{2 R}+\overline{b_{3 R}}\left(-\frac{1}{2}-s_{W}^{2} Q_{b}\right) b_{3 R}+ \\
& {\left.\left[\overline{b_{2 R}}\left(-\frac{1}{2}\right) b_{3 R}+\text { h.c. }\right]\right\} Z }
\end{aligned}
$$

where $g_{Z} \equiv \sqrt{g_{L}^{2}+g^{\prime 2}}, Q_{b}=-1 / 3$. Note that the $b_{1} b_{1} Z$ interactions come out standard due to the custodial protection. The photon couplings are not shown and as usual has vectorlike couplings to the fermions given by their electromagnetic charge. We have taken 
all $\mathcal{I}_{\psi \psi V}=1$ as earlier, ignoring corrections to this due to EWSB (0) - (1) gauge boson mixing which are at most a few percent. The Higgs interactions are got by $v \rightarrow v(1+h / v)$ and are

$$
\mathcal{L}^{h} \supset \frac{1}{\sqrt{1+2 r_{b}^{2}}}\left(\frac{2 m^{b b^{\prime}}}{v}\right)\left[\overline{b_{1 L}} b_{3 R}-\sqrt{2} r_{b} \overline{b_{3 L}} b_{3 R}\right] h+\text { h.c. }
$$

\section{C $\quad \chi$ signature in more detail}

In this section, we perform a more detailed analysis of the $p p \rightarrow \chi_{1} t W \rightarrow t W t W$ channel that we discussed in section 5.1. Our aim is to show that the discovery luminosity estimates that we obtained there stand up to a more detailed analysis. In section 5.1, to estimate the LHC discovery reach of $\chi$, we compute the $p p \rightarrow t t W W \rightarrow t t W \ell \nu$ as the SM background for $p p \rightarrow \chi_{1} t W \rightarrow t W t \ell \nu$. For $M_{\chi} \gtrsim 750 \mathrm{GeV}$, the top quarks will be quite boosted and so, instead of using conventional top reconstruction algorithm with $b$-tagging, one could use modern top-tagging algorithms [83-85] like HEPTopTagger [83] which has much higher top-tagging efficiency. These advanced algorithms can achieve a reconstruction efficiency $\epsilon_{t} \sim 40-50 \%$ (mistag rate is only a few percent and can even be reduced further) in the top- $p_{T}$ ranging from $200 \mathrm{GeV}$ to $600 \mathrm{GeV}$. With HEPTopTagger, $b$-tagging is not necessary and combinatorics issues are automatically resolved by the algorithm. We note that the hadronic $W$-tagging efficiency is also quite high. It is around $70-80 \%$ for moderately boosted $W[86,87]$.

With these in mind, after reconstruction of the two high $p_{T}$ tops $\left(p_{T} \geq 200 \mathrm{GeV}\right)$, for the $p p \rightarrow \chi_{1} t W \rightarrow t t W \ell \nu$ signal process, a problematic background can be the SM $p p \rightarrow t t j j \ell \nu$. The main contribution for this background will come from the processes where the jets are from the decay of $Z$ or $W$, or two QCD jets. We demonstrate here that these extra backgrounds can be brought under control, for example by using the following

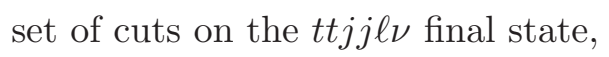

- Cut-II:

1. $|y(l)|,|y(j)| \leq 2.5, \quad p_{T}(l), p_{T}(j) \geq 25 \mathrm{GeV}$

2. $p_{T}(t) \geq 200 \mathrm{GeV}$,

3. $\left|M(j j)-M_{W}\right| \leq 15 \mathrm{GeV}$,

4. $\left(\left|M\left(t_{1} j j\right)-M_{\chi_{1}}\right|\right.$ or $\left.\left|M\left(t_{2} j j\right)-M_{\chi_{1}}\right|\right) \leq 0.2 M_{\chi_{1}}$ where $t_{1}$ and $t_{2}$ are the two $p_{T}$-ordered tops.

In table 12 we display the signal and total background cross-sections with Cut-II for the $\chi$ benchmark points. Here the background includes all the processes where the jets are coming from a EW vector boson or are QCD jets. From the table 12 we can see that Cut-II is very effective to reduce background for higher $M_{\chi}$ values and thus, making the $\chi$ discovery channel signal rate limited for all benchmark $M_{\chi}$ values we have considered. The luminosity requirements obtained here differ from the ones shown in table 6 by about $10-15 \%$ only. 


\begin{tabular}{|c|c|c|c|c|}
\hline \multirow{2}{*}{$\begin{array}{c}M_{\chi} \\
(\mathrm{GeV})\end{array}$} & \multicolumn{3}{|c|}{$\sigma(\mathrm{fb})$ after Cut-II } & $\mathcal{L}_{D}$ \\
\cline { 2 - 4 } & Signal & $t t j j \ell \nu(\mathrm{EW} \mathrm{BG})$ & $t t j j \ell \nu(\mathrm{QCD} \mathrm{BG})$ & $\mathrm{fb}^{-1}$ \\
\hline 500 & 136.33 & 0.18 & 0.41 & 0.654 \\
\hline 750 & 33.66 & 0.16 & 0.29 & 2.647 \\
\hline 1000 & 8.006 & 0.09 & 0.18 & 11.13 \\
\hline 1250 & 2.173 & 0.05 & 0.10 & 41.01 \\
\hline 1500 & 0.660 & 0.03 & 0.05 & 135.0 \\
\hline 1750 & 0.217 & 0.02 & 0.03 & 410.6 \\
\hline
\end{tabular}

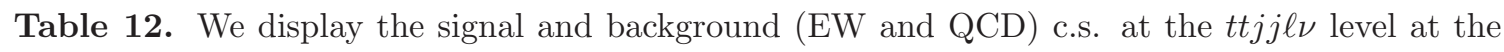
$14 \mathrm{TeV}$ LHC after Cut-II as defined in the text. While computing $\mathcal{L}_{D}$ we multiply both signal and background by a factor $\eta=\left(\epsilon_{t}\right)^{2} \times\left(B R_{W \rightarrow j j}\right)^{2}$. We use $B R_{W \rightarrow j j}=0.67$ and, take $\epsilon_{t}=0.5$.

Open Access. This article is distributed under the terms of the Creative Commons Attribution License (CC-BY 4.0), which permits any use, distribution and reproduction in any medium, provided the original author(s) and source are credited.

\section{References}

[1] S. Gopalakrishna, T. Mandal, S. Mitra and R. Tibrewala, LHC Signatures of a Vector-like b', Phys. Rev. D 84 (2011) 055001 [arXiv: 1107.4306] [INSPIRE].

[2] R. Contino, L. Da Rold and A. Pomarol, Light custodians in natural composite Higgs models, Phys. Rev. D 75 (2007) 055014 [hep-ph/0612048] [INSPIRE].

[3] C. Anastasiou, E. Furlan and J. Santiago, Realistic Composite Higgs Models, Phys. Rev. D 79 (2009) 075003 [arXiv:0901.2117] [INSPIRE].

[4] N. Vignaroli, Discovering the composite Higgs through the decay of a heavy fermion, JHEP 07 (2012) 158 [arXiv:1204.0468] [INSPIRE].

[5] A. De Simone, O. Matsedonskyi, R. Rattazzi and A. Wulzer, A First Top Partner Hunter's Guide, JHEP 04 (2013) 004 [arXiv:1211.5663] [INSPIRE].

[6] T. Han, H.E. Logan, B. McElrath and L.-T. Wang, Phenomenology of the little Higgs model, Phys. Rev. D 67 (2003) 095004 [hep-ph/0301040] [INSPIRE].

[7] M.S. Carena, J. Hubisz, M. Perelstein and P. Verdier, Collider signature of T-quarks, Phys. Rev. D 75 (2007) 091701 [hep-ph/0610156] [INSPIRE].

[8] S. Matsumoto, T. Moroi and K. Tobe, Testing the Littlest Higgs Model with T-parity at the Large Hadron Collider, Phys. Rev. D 78 (2008) 055018 [arXiv: 0806.3837] [inSPIRE].

[9] J. Berger, J. Hubisz and M. Perelstein, A Fermionic Top Partner: Naturalness and the LHC, JHEP 07 (2012) 016 [arXiv:1205.0013] [INSPIRE].

[10] J. Kang, P. Langacker and B.D. Nelson, Theory and Phenomenology of Exotic Isosinglet Quarks and Squarks, Phys. Rev. D 77 (2008) 035003 [arXiv:0708.2701] [INSPIRE].

[11] P.W. Graham, A. Ismail, S. Rajendran and P. Saraswat, A Little Solution to the Little Hierarchy Problem: A Vector-like Generation, Phys. Rev. D 81 (2010) 055016 [arXiv:0910.3020] [INSPIRE]. 
[12] S.P. Martin, Raising the Higgs mass with Yukawa couplings for isotriplets in vector-like extensions of minimal supersymmetry, Phys. Rev. D 82 (2010) 055019 [arXiv:1006.4186] [INSPIRE].

[13] T. Li, Z. Murdock, S. Nandi and S.K. Rai, Quark lepton unification in higher dimensions, Phys. Rev. D 85 (2012) 076010 [arXiv: 1201.5616] [InSPIRE].

[14] F. del Aguila, L. Ametller, G.L. Kane and J. Vidal, Vector Like Fermion and Standard Higgs Production at Hadron Colliders, Nucl. Phys. B 334 (1990) 1 [InSPIRE].

[15] F. del Aguila, G.L. Kane and M. Quirós, A Possible Method to Produce and Detect Higgs Bosons at Hadron Colliders, Phys. Rev. Lett. 63 (1989) 942 [INSPIRE].

[16] A. Djouadi and G. Moreau, Higgs production at the LHC in warped extra-dimensional models, Phys. Lett. B 660 (2008) 67 [arXiv:0707.3800] [INSPIRE].

[17] A. Azatov et al., Higgs boson production via vector-like top-partner decays: Diphoton or multilepton plus multijets channels at the LHC, Phys. Rev. D 85 (2012) 115022 [arXiv: 1204.0455] [INSPIRE].

[18] C. Bouchart and G. Moreau, Higgs boson phenomenology and VEV shift in the RS scenario, Phys. Rev. D 80 (2009) 095022 [arXiv:0909.4812] [INSPIRE].

[19] ATLAS collaboration, Observation of a new particle in the search for the Standard Model Higgs boson with the ATLAS detector at the LHC, Phys. Lett. B 716 (2012) 1 [arXiv: 1207.7214] [INSPIRE].

[20] CMS collaboration, Observation of a new boson at a mass of $125 \mathrm{GeV}$ with the CMS experiment at the LHC, Phys. Lett. B 716 (2012) 30 [arXiv:1207.7235] [InSPIRE].

[21] G. Moreau, Constraining extra-fermion(s) from the Higgs boson data, Phys. Rev. D 87 (2013) 015027 [arXiv: 1210.3977] [INSPIRE].

[22] N. Bonne and G. Moreau, Reproducing the Higgs boson data with vector-like quarks, Phys. Lett. B 717 (2012) 409 [arXiv:1206.3360] [INSPIRE].

[23] A. Djouadi, G. Moreau and F. Richard, Forward-backward asymmetries of the bottom and top quarks in warped extra-dimensional models: LHC predictions from the LEP and Tevatron anomalies, Phys. Lett. B 701 (2011) 458 [arXiv: 1105.3158] [INSPIRE].

[24] A. Djouadi, G. Moreau, F. Richard and R.K. Singh, The forward-backward asymmetry of top quark production at the Tevatron in warped extra dimensional models, Phys. Rev. D 82 (2010) 071702 [arXiv:0906.0604] [INSPIRE].

[25] A. Djouadi, G. Moreau and F. Richard, Resolving the $A_{F B}^{b}$ puzzle in an extra dimensional model with an extended gauge structure, Nucl. Phys. B 773 (2007) 43 [hep-ph/0610173] [INSPIRE].

[26] C. Bouchart and G. Moreau, The precision electroweak data in warped extra-dimension models, Nucl. Phys. B 810 (2009) 66 [arXiv:0807.4461] [InSPIRE].

[27] F. del Aguila, J.A. Aguilar-Saavedra and R. Miquel, Constraints on top couplings in models with exotic quarks, Phys. Rev. Lett. 82 (1999) 1628 [hep-ph/9808400] [INSPIRE].

[28] F. del Aguila, M. Pérez-Victoria and J. Santiago, Observable contributions of new exotic quarks to quark mixing, JHEP 09 (2000) 011 [hep-ph/0007316] [INSPIRE].

[29] J.A. Aguilar-Saavedra, Effects of mixing with quark singlets, Phys. Rev. D 67 (2003) 035003 [Erratum ibid. D 69 (2004) 099901] [hep-ph/0210112] [INSPIRE]. 
[30] G. Cacciapaglia, A. Deandrea, D. Harada and Y. Okada, Bounds and Decays of New Heavy Vector-like Top Partners, JHEP 11 (2010) 159 [arXiv:1007.2933] [INSPIRE].

[31] G. Cacciapaglia, A. Deandrea, L. Panizzi, N. Gaur, D. Harada and Y. Okada, Heavy Vector-like Top Partners at the LHC and flavour constraints, JHEP 03 (2012) 070 [arXiv:1108.6329] [INSPIRE].

[32] Y. Okada and L. Panizzi, LHC signatures of vector-like quarks, Adv. High Energy Phys. 2013 (2013) 364936 [arXiv: 1207.5607] [INSPIRE].

[33] J.A. Aguilar-Saavedra, R. Benbrik, S. Heinemeyer and M. Pérez-Victoria, Handbook of vectorlike quarks: Mixing and single production, Phys. Rev. D 88 (2013) 094010 [arXiv: 1306.0572] [INSPIRE].

[34] R. Contino and G. Servant, Discovering the top partners at the LHC using same-sign dilepton final states, JHEP 06 (2008) 026 [arXiv:0801.1679] [INSPIRE].

[35] J. Mrazek and A. Wulzer, A Strong Sector at the LHC: Top Partners in Same-Sign Dileptons, Phys. Rev. D 81 (2010) 075006 [arXiv:0909.3977] [InSPIRE].

[36] C. Dennis, M. Karagoz, G. Servant and J. Tseng, Multi-W events at LHC from a warped extra dimension with custodial symmetry, hep-ph/0701158 [INSPIRE].

[37] G. Cacciapaglia, A. Deandrea, L. Panizzi, S. Perries and V. Sordini, Heavy Vector-like quark with charge 5/3 at the LHC, JHEP 03 (2013) 004 [arXiv:1211.4034] [INSPIRE].

[38] J.A. Aguilar-Saavedra, Pair production of heavy $Q=2 / 3$ singlets at $L H C$, Phys. Lett. B 625 (2005) 234 [Erratum ibid. B 633 (2006) 792-793] [hep-ph/0506187] [INSPIRE].

[39] J.A. Aguilar-Saavedra, Light Higgs boson discovery from fermion mixing, JHEP 12 (2006) 033 [hep-ph/0603200] [INSPIRE].

[40] A. Atre, M. Chala and J. Santiago, Searches for New Vector Like Quarks: Higgs Channels, JHEP 05 (2013) 099 [arXiv: 1302.0270] [INSPIRE].

[41] K. Harigaya, S. Matsumoto, M.M. Nojiri and K. Tobioka, Search for the Top Partner at the LHC using Multi-b-Jet Channels, Phys. Rev. D 86 (2012) 015005 [arXiv:1204.2317] [INSPIRE].

[42] J.A. Aguilar-Saavedra, Identifying top partners at LHC, JHEP 11 (2009) 030 [arXiv:0907.3155] [INSPIRE].

[43] M. Carena, A.D. Medina, B. Panes, N.R. Shah and C.E.M. Wagner, Collider phenomenology of gauge-Higgs unification scenarios in warped extra dimensions, Phys. Rev. D 77 (2008) 076003 [arXiv: 0712.0095] [inSPIRE].

[44] R. Barcelo, A. Carmona, M. Chala, M. Masip and J. Santiago, Single Vectorlike Quark Production at the LHC, Nucl. Phys. B 857 (2012) 172 [arXiv:1110.5914] [InSPIRE].

[45] C. Bini, R. Contino and N. Vignaroli, Heavy-light decay topologies as a new strategy to discover a heavy gluon, JHEP 01 (2012) 157 [arXiv:1110.6058] [INSPIRE].

[46] A. Atre, M. Carena, T. Han and J. Santiago, Heavy Quarks Above the Top at the Tevatron, Phys. Rev. D 79 (2009) 054018 [arXiv:0806.3966] [InSPIRE].

[47] T. Han, I. Lewis and Z. Liu, Colored Resonant Signals at the LHC: Largest Rate and Simplest Topology, JHEP 12 (2010) 085 [arXiv: 1010.4309] [INSPIRE]. 
[48] A. Atre, G. Azuelos, M. Carena, T. Han, E. Ozcan et al., Model-Independent Searches for New Quarks at the LHC, JHEP 08 (2011) 080 [arXiv:1102.1987] [INSPIRE].

[49] M. Buchkremer, G. Cacciapaglia, A. Deandrea and L. Panizzi, Model Independent Framework for Searches of Top Partners, Nucl. Phys. B 876 (2013) 376 [arXiv:1305.4172] [InSPIRE].

[50] New Physics Working Group collaboration, G. Brooijmans et al., New Physics at the LHC. A Les Houches Report: Physics at TeV Colliders 2009 - New Physics Working Group, arXiv: 1005.1229 [INSPIRE].

[51] S. Gopalakrishna, T. Mandal, S. Mitra and R. Tibrewala, Phenomenology of warped-space custodian b', Contribution 4, pg. 719 in: D.K. Ghosh et al., Working group report: Physics at the Large Hadron Collider, Pramana 76 (2011) 707.

[52] A. Alves, E. Ramirez Barreto, D.A. Camargo and A.G. Dias, A Model with Chiral Quarks of Electric Charges -4/3 and 5/3, JHEP 07 (2013) 129 [arXiv:1306.1275] [INSPIRE].

[53] ATLAS collaboration, Search for pair production of new heavy quarks that decay to a $\mathbf{Z}$ boson and a third generation quark in $\mathbf{p p}$ collisions at $\sqrt{\mathbf{s}}=\mathbf{8} \mathrm{TeV}$ with the ATLAS detector, ATLAS-CONF-2013-056 (2013).

[54] ATLAS collaboration, Search for Single Production of Vector-like Quarks Coupling to Light Generations in 4.64 ifb of Data at sqrts $=7 \mathrm{TeV}$, ATLAS-CONF-2012-137 (2012).

[55] CMS collaboration, Search for a heavy partner of the top quark with charge 5/3, CMS-PAS-B2G-12-003.

[56] CMS collaboration, Search for T5/3 top partners in same-sign dilepton final state, CMS-PAS-B2G-12-012.

[57] ATLAS collaboration, Search for pair production of heavy top-like quarks decaying to a high- $p_{\mathrm{T}} W$ boson and $a b$ quark in the lepton plus jets final state in pp collisions at $\sqrt{s}=8 \mathrm{TeV}$ with the ATLAS detector, ATLAS-CONF-2013-060 (2013).

[58] L. Randall and R. Sundrum, A large mass hierarchy from a small extra dimension, Phys. Rev. Lett. 83 (1999) 3370 [hep-ph/9905221] [INSPIRE].

[59] J.M. Maldacena, The large- $N$ limit of superconformal field theories and supergravity, Adv. Theor. Math. Phys. 2 (1998) 231 [Int. J. Theor. Phys. 38 (1999) 1113] [hep-th/9711200] [INSPIRE].

[60] Y. Grossman and M. Neubert, Neutrino masses and mixings in nonfactorizable geometry, Phys. Lett. B 474 (2000) 361 [hep-ph/9912408] [INSPIRE].

[61] T. Gherghetta and A. Pomarol, Bulk fields and supersymmetry in a slice of AdS, Nucl. Phys. B 586 (2000) 141 [hep-ph/0003129] [INSPIRE].

[62] K. Agashe, A. Delgado, M.J. May and R. Sundrum, RS1, custodial isospin and precision tests, JHEP 08 (2003) 050 [hep-ph/0308036] [INSPIRE].

[63] K. Agashe, R. Contino, L. Da Rold and A. Pomarol, A custodial symmetry for Z $b \bar{b}$, Phys. Lett. B 641 (2006) 62 [hep-ph/0605341] [INSPIRE].

[64] K.-w. Choi and I.-W. Kim, One loop gauge couplings in $A d S_{5}$, Phys. Rev. D 67 (2003) 045005 [hep-th/0208071] [INSPIRE].

[65] K. Agashe and G. Servant, Baryon number in warped GUTs: Model building and (dark matter related) phenomenology, JCAP 02 (2005) 002 [hep-ph/0411254] [INSPIRE]. 
[66] K. Agashe, G. Perez and A. Soni, Flavor structure of warped extra dimension models, Phys. Rev. D 71 (2005) 016002 [hep-ph/0408134] [INSPIRE].

[67] H. Davoudiasl, J.L. Hewett and T.G. Rizzo, Phenomenology of the Randall-Sundrum Gauge Hierarchy Model, Phys. Rev. Lett. 84 (2000) 2080 [hep-ph/9909255] [INSPIRE].

[68] K. Agashe, A. Belyaev, T. Krupovnickas, G. Perez and J. Virzi, LHC Signals from Warped Extra Dimensions, Phys. Rev. D 77 (2008) 015003 [hep-ph/0612015] [INSPIRE].

[69] B. Lillie, L. Randall and L.-T. Wang, The bulk RS KK-gluon at the LHC, JHEP 09 (2007) 074 [hep-ph/0701166] [INSPIRE].

[70] K. Agashe et al., LHC Signals for Warped Electroweak Neutral Gauge Bosons, Phys. Rev. D 76 (2007) 115015 [arXiv: 0709.0007] [inSPIRE].

[71] A. Djouadi, G. Moreau and R.K. Singh, Kaluza-Klein excitations of gauge bosons at the LHC, Nucl. Phys. B 797 (2008) 1 [arXiv:0706.4191] [INSPIRE].

[72] K. Agashe, S. Gopalakrishna, T. Han, G.-Y. Huang and A. Soni, LHC Signals for Warped Electroweak Charged Gauge Bosons, Phys. Rev. D 80 (2009) 075007 [arXiv:0810.1497] [INSPIRE].

[73] F. Ledroit, G. Moreau and J. Morel, Probing RS scenarios of flavour at LHC via leptonic channels, JHEP 09 (2007) 071 [hep-ph/0703262] [inSPIRE].

[74] H. Davoudiasl, S. Gopalakrishna, E. Ponton and J. Santiago, Warped 5-Dimensional Models: Phenomenological Status and Experimental Prospects, New J. Phys. 12 (2010) 075011 [arXiv:0908.1968] [INSPIRE].

[75] G. Moreau and J.I. Silva-Marcos, Flavor physics of the RS model with KK masses reachable at LHC, JHEP 03 (2006) 090 [hep-ph/0602155] [INSPIRE].

[76] G. Moreau and J.I. Silva-Marcos, Neutrinos in warped extra dimensions, JHEP 01 (2006) 048 [hep-ph/0507145] [INSPIRE].

[77] Particle Data Group collaboration, J. Beringer et al., Review of Particle Physics (RPP), Phys. Rev. D 86 (2012) 010001 [INSPIRE].

[78] N.D. Christensen and C. Duhr, FeynRules - Feynman rules made easy, Comput. Phys. Commun. 180 (2009) 1614 [arXiv:0806.4194] [INSPIRE].

[79] J. Alwall, M. Herquet, F. Maltoni, O. Mattelaer and T. Stelzer, MadGraph 5: Going Beyond, JHEP 06 (2011) 128 [arXiv:1106.0522] [INSPIRE].

[80] J. Pumplin, D.R. Stump, J. Huston, H.L. Lai, P.M. Nadolsky and W.K. Tung, New generation of parton distributions with uncertainties from global QCD analysis, JHEP 07 (2002) 012 [hep-ph/0201195] [INSPIRE].

[81] B. Coleppa, T. Mandal and S. Mitra, Coupling Extraction From Off-Shell Cross-sections, arXiv: 1401.4039 [INSPIRE].

[82] A. Girdhar, A clean signal for a top-like isosinglet fermion at the Large Hadron Collider, Pramana 81 (2013) 975 [arXiv: 1204.2885] [INSPIRE].

[83] T. Plehn, M. Spannowsky, M. Takeuchi and D. Zerwas, Stop Reconstruction with Tagged Tops, JHEP 10 (2010) 078 [arXiv:1006.2833] [INSPIRE].

[84] T. Plehn, G.P. Salam and M. Spannowsky, Fat Jets for a Light Higgs, Phys. Rev. Lett. 104 (2010) 111801 [arXiv:0910.5472] [INSPIRE]. 
[85] T. Plehn and M. Spannowsky, Top Tagging, J. Phys. G 39 (2012) 083001 [arXiv:1112.4441] [INSPIRE].

[86] J. Thaler and K. Van Tilburg, Identifying Boosted Objects with $\mathrm{N}$-subjettiness, JHEP 03 (2011) 015 [arXiv: 1011.2268] [INSPIRE].

[87] CMS Collaboration, Identifying Hadronically Decaying Vector Bosons Merged into a Single Jet, CMS-PAS-JME-13-006. 